\title{
Improving the Complexity of the Lorenz Dynamics
}

\author{
María Pilar Mareca and Borja Bordel \\ Department of Physic Electronics, Universidad Politécnica de Madrid, Avenida Complutense No. 30, 28040 Madrid, Spain
}

Correspondence should be addressed to María Pilar Mareca; mpmareca@fis.upm.es

Received 26 July 2016; Accepted 19 September 2016; Published 10 January 2017

Academic Editor: Michael Small

Copyright (C) 2017 M. P. Mareca and B. Bordel. This is an open access article distributed under the Creative Commons Attribution License, which permits unrestricted use, distribution, and reproduction in any medium, provided the original work is properly cited.

\begin{abstract}
A new four-dimensional, hyperchaotic dynamic system, based on Lorenz dynamics, is presented. Besides, the most representative dynamics which may be found in this new system are located in the phase space and are analyzed here. The new system is especially designed to improve the complexity of Lorenz dynamics, which, despite being a paradigm to understand the chaotic dissipative flows, is a very simple example and shows great vulnerability when used in secure communications. Here, we demonstrate the vulnerability of the Lorenz system in a general way. The proposed $4 \mathrm{D}$ system increases the complexity of the Lorenz dynamics. The trajectories of the novel system include structures going from chaos to hyperchaos and chaotic-transient solutions. The symmetry and the stability of the proposed system are also studied. First return maps, Poincaré sections, and bifurcation diagrams allow characterizing the global system behavior and locating some coexisting structures. Numerical results about the first return maps, Poincaré cross sections, Lyapunov spectrum, and Kaplan-Yorke dimension demonstrate the complexity of the proposed equations.
\end{abstract}

\section{Introduction}

A chaotic system is a highly sensitive nonlinear system. The main characteristic of chaos is the sensibility to the initial conditions. This sensibility is sometimes known as "butterfly effect" in honor of Lorenz [1] who described in 1963 the first natural phenomenon showing a chaotic behavior (in this case, the atmospheric evolution) being represented by a differential system (1) which generates a trajectory in the phase space similar to a butterfly (see Figure 1):

$$
\begin{aligned}
& \dot{x}=\sigma(y-x), \\
& \dot{y}=\rho x-y-x z, \\
& \dot{z}=x y-\beta z .
\end{aligned}
$$

The Lorenz system has been extensively employed: cyphers [2,3], circuits [4], engineered systems [5], and so forth have been based on this dynamics. However, despite being the first described chaotic system and, probably, the most used and the most popular one, it presents a complexity degree pretty low. For example, as we will see in Section 2, some encryptions based on Lorenz system may be broken by synchronizing the cypher with an external system (although its parameters were unknown). The underlying cause of this weakness is the great amount of redundant information present in the components of their dynamics that it is precisely associated with the low degree of complexity of the system. Even more, some works have shown that it is possible to recover the three systems' components from only one of them [6].

In order to increase its degree of complexity, several works have tried to modify the Lorenz dynamics. Some of them try to increase the number of fixed points in the system [7]. Others are focused on including commutated terms which modify the number of wings in Lorenz's attractor [8]. Some articles including new nonlinearities in the Lorenz system to improve its complexity can be also found [9]. As an extreme case, techniques to build $m \times n$ Lorenz-like attractors have been also reported [10].

Among all these previous works, a very important group of articles are those focused on the creation of Lorenz-like "hyperchaotic systems." Traditionally, hyperchaos was defined [11] as a chaotic behavior characterized for more than one positive Lyapunov exponent; that is, the dynamics expand in more than one direction (therefore, hyperchaos can only be found in high order systems). However, in recent proposals, hyperchaos has been also applied to high-order 


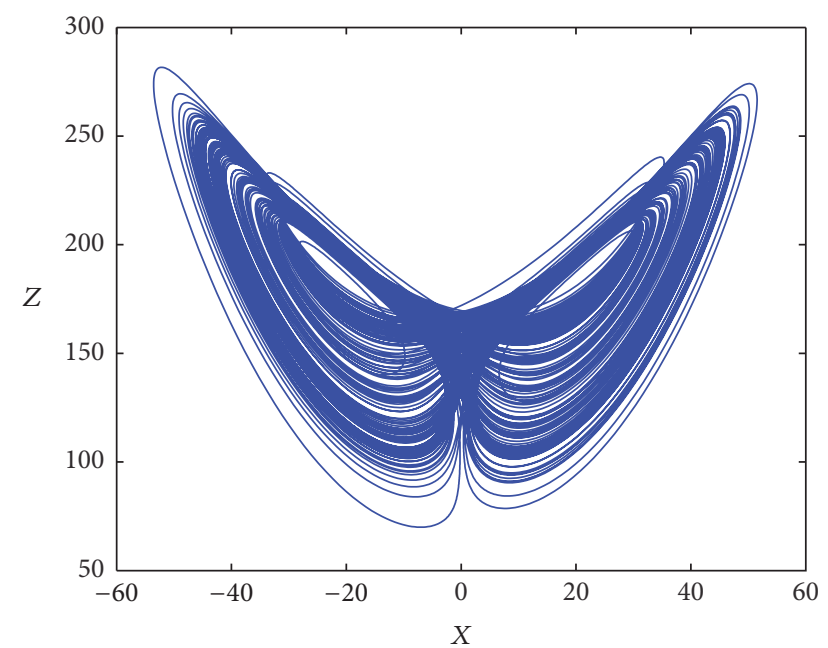

FIGURE 1: $X Z$ composition of the Lorenz system solution for $\{\sigma=10$, $\rho=178$, and $\beta=8 / 3\}$.

systems which present only one positive Lyapunov exponent [12], besides the zero exponent, but whose value is much higher than the common values (e.g., for Lorenz system, the maximum value of the positive Lyapunov exponent is around $\lambda_{\max }=5.2$ when $\{\sigma=25, \rho=420$, and $\beta=8 / 3\}$ and exhibits a Kaplan-Yorke dimension of $D_{\mathrm{KY}}=2.17$ ). There have been proposed many hyperchaotic systems, with some designed to facilitate the electronic implementation [13] and others designed to create certain topologies in the phase space [14] and, even, new hyperchaotic dynamics have been described to be employed in different encryption schemes [15], with most of them being based on Lorenz equations [1618]. Nevertheless, these proposals do not cover the objective of increasing the complexity at all. While these new dynamics are more complex, that increased complexity is further supported by the fact of having at least one additional equation instead of the appearance of trajectories whose entropy is higher. Actually, the value of the maximum Lyapunov exponent in these cases is around $\lambda_{\max }=2.4$ and the rate of contraction is high, obtaining, thus, weak hyperchaos.

Therefore, the aim of this article is to describe a new hyperchaotic system, being able to generate highly complex and novel structures. For that, we seek to strengthen at least one direction of expansion and reduce the rate of contraction (but they should remain dominant in order to preserve the system as dissipative).

The rest of the paper is organized as follows. In the next section, a study about the redundant data in the components of Lorenz's dynamics is presented. In Section 3, the new hyperchaotic system is introduced and its basic topological properties are studied. In Section 4, the regular structures are located and analyzed. In Section 5, chaotic attractors are described. In Section 6, complex chaotic and hyperchaotic trajectories are analyzed, describing the special case of the chaotic-transient solutions. Section 7 concludes the paper.

\section{Insecurity in Lorenz-Based Cyphers}

It has been proven in many articles that Lorenz system can be self-synchronized [4]. In particular, some of the components of the dynamics contain so much information about the flow (values of the parameters, topology being generated, etc.) in which the most weak synchronization schemes are useful when applied to the Lorenz dynamics. For example, even active-passive decomposition schemes [19], where only one of the terms in one of the equations is modified (in contrast to other schemes [20], where one or various equations are totally removed), allow fast synchronization.

Theorem 1. Consider two Lorenz systems (2), coupled using an active-passive decomposition scheme. If enough amount of time is elapsed, then both systems get completely synchronized (in amplitude and phase):

$$
\begin{aligned}
& \dot{x}_{e}=\sigma_{0}\left(y_{e}-x_{e}\right), \\
& \dot{y}_{e}=\rho_{0} x_{e}-y_{e}-x_{e} z_{e}, \\
& \dot{z}_{e}=x_{e} y_{e}-\beta_{0} z_{e}, \\
& \dot{x}_{r}=\sigma_{0}\left(y_{e}-x_{r}\right), \\
& \dot{y}_{r}=\rho_{0} x_{r}-y_{r}-x_{r} z_{r}, \\
& \dot{z}_{r}=x_{r} y_{r}-\beta_{0} z_{r} .
\end{aligned}
$$

Proof. A new function, called error function, is defined as follows:

$$
\vec{e}=\left(e_{1}, e_{2}, e_{3}\right)=\left(x_{r}, y_{r}, z_{r}\right)-\left(x_{e}, y_{e}, z_{e}\right) .
$$

Thus, both coupled Lorenz systems get synchronized in amplitude and phase if $\vec{e} \rightarrow \overrightarrow{0}$ when $t \rightarrow \infty$. The error function satisfies the following differential system:

$$
\begin{aligned}
& \dot{e_{1}}=-\sigma_{0} e_{1}, \\
& \dot{e_{2}}=\rho_{0} e_{1}-e_{2}-x_{r} z_{r}+x_{e} z_{e}, \\
& \dot{e_{3}}=x_{r} y_{r}-x_{e} y_{e}-\beta_{0} e_{3} .
\end{aligned}
$$

The first differential equation is decoupled, so it may be solved directly (5). Considering $\sigma_{0}>0$ (due to its physical meaning), $e_{1}$ tends to zero (6) and the limit condition $x_{r} \rightarrow$ $x_{e}$ is satisfied:

$$
\begin{aligned}
e_{1}(t) & =K e^{-\sigma_{0} t}, \\
\lim _{t \rightarrow \infty} e_{1} & =\lim _{t \rightarrow \infty} K e^{-\sigma_{0} t}=0 .
\end{aligned}
$$

Then, the resulting differential system (7) at $t \rightarrow \infty$ may be used to prove the stability of the rest of the components:

$$
\begin{aligned}
& e_{1}=0, \\
& \dot{e}_{2}=-e_{2}-x_{e} e_{3}, \\
& \dot{e_{3}}=x_{e} e_{2}-\beta_{0} e_{3} .
\end{aligned}
$$


A Lyapunov function satisfying two conditions is proposed as follows:

$$
L(\vec{e})=\frac{1}{2} \vec{e} \cdot \vec{e} .
$$

(1) $L(\vec{e})$ presents a strict minimum at the origin, so that $L(\overrightarrow{0})=0$ and $L(\vec{e})>0 \forall \vec{e} \neq \overrightarrow{0}$.

(2) $d L(\vec{e}) / d t<0$ in any reduced neighborhood of the origin. In fact, $d L(\vec{e}) / d t=\vec{e} \cdot \dot{\vec{e}}=e_{2} \dot{e_{2}}+e_{3} \dot{e_{3}}=e_{2}\left(-e_{2}-\right.$ $\left.x_{e} e_{3}\right)+e_{3}\left(x_{e} e_{2}-\beta_{0} e_{3}\right)=-e_{2} e_{2}-\beta_{0} e_{3} e_{3} \forall \beta_{0}>0$.

If $\beta_{0}>0$, then Lyapunov's theorem about the asymptotic stability guarantees that $\lim _{t \rightarrow \infty} \vec{e}=\overrightarrow{0}$ and then $\left(x_{r}, y_{r}, z_{r}\right) \rightarrow$ $\left(x_{e}, y_{e}, z_{e}\right)$ when $t \rightarrow \infty$.

Theorem 1, in fact, enables the creation of cryptographic systems based on self-synchronized Lorenz system containing encrypted information. However, Theorem 1 might be understood in another way. If so much information is carried in each one of Lorenz's system components, are Lorenz-based cryptosystems safe?

Theorem 2. The information about the system in any of the Lorenz dynamic components allows any intruder system (9) not only to synchronize the temporal evolution but also to obtain the values of the employed parameters in the transmitter:

$$
\begin{aligned}
& \dot{x}_{r}=\sigma_{0}\left(y_{e}-x_{r}\right), \\
& \dot{y}_{r}=\rho(t) x_{r}-y_{r}-x_{r} z_{r}, \\
& \dot{z}_{r}=x_{r} y_{r}-\beta(t) z_{r} .
\end{aligned}
$$

Proof. Two first differential equations (10) determining the evolution laws of $\rho(t)$ and $\beta(t)$ are defined:

$$
\begin{aligned}
& \dot{\rho}(t)=-e_{2} x_{r}, \\
& \dot{\beta}(t)=e_{3} z_{r} .
\end{aligned}
$$

Besides, both error functions for the temporal evolution of the systems and for the parameters values are also defined as follows:

$$
\begin{aligned}
& \vec{e}=\left(x_{r}, y_{r}, z_{r}\right)-\left(x_{e}, y_{e}, z_{e}\right), \\
& \vec{d}=(\rho(t), \beta(t))-\left(\rho_{0}, \beta_{0}\right) .
\end{aligned}
$$

If it is satisfied that $\vec{e} \rightarrow \overrightarrow{0}$ and $\vec{d} \rightarrow \overrightarrow{0}$ when $t \rightarrow \infty$; then Theorem 2 is verified. For that, the expressions of the differential systems for the error functions are deduced:

$$
\begin{aligned}
& \dot{\vec{e}}=\left(\begin{array}{c}
-\sigma_{0} e_{1} \\
\rho(t) x_{r}-\rho_{0} x_{e}-e_{2}-x_{r} z_{r}+x_{e} y_{e} \\
x_{r} y_{r}-\beta(t) z_{r}-x_{e} y_{e}+\beta_{0} z_{e}
\end{array}\right), \\
& \dot{\vec{d}}=\left(\begin{array}{c}
-e_{2} x_{r} \\
e_{3} z_{r}
\end{array}\right) .
\end{aligned}
$$

As it is said previously, the first equation is decoupled and it can be proven that $e_{1}$ tends to zero when $t \rightarrow \infty$. Then, when $t \rightarrow \infty$, the differential system for the error functions may be simplified:

$$
\begin{aligned}
& \dot{\vec{e}}=\left(\begin{array}{c}
-\sigma_{0} e_{1} \\
d_{1} x_{e}-e_{2}-x_{e} e_{3} \\
-\beta(t) z_{r}+x_{e} e_{2}+\beta_{0} z_{e}
\end{array}\right), \\
& \dot{\vec{d}}=\left(\begin{array}{c}
-e_{2} x_{e} \\
e_{3} z_{r}
\end{array}\right) .
\end{aligned}
$$

A Lyapunov function is proposed (14), satisfying two conditions:

$$
L(\vec{e}, \vec{d})=\frac{1}{2}(\vec{e} \cdot \vec{e}+\vec{d} \cdot \vec{d}) .
$$

(1) $L(\vec{e}, \vec{d})$ presents a strict minimum at the origin, so that $L(\overrightarrow{0}, \overrightarrow{0})=0$ and $L(\vec{e}, \vec{d})>0 \forall \vec{e}, \vec{d} \neq \overrightarrow{0}$.

(2) $L(\vec{e}, \vec{d}) / d t<0$ in any neighborhood of the origin. In fact, $d L(\vec{e}, \vec{d}) / d t=\vec{e} \cdot \dot{\vec{e}}+\vec{d} \cdot \dot{\vec{d}}=e_{2} \dot{e}_{2}+e_{3} \dot{e}_{3}+d_{1} \dot{d}_{1}+$ $d_{2} \dot{d}_{2}=e_{2}\left(d_{1} x_{e}-e_{2}-x_{e} e_{3}\right)+e_{3}\left(-\beta(t) z_{r}+x_{e} e_{2}+\beta_{0} z_{e}\right)+$ $-e_{2} x_{e} d_{1}+e_{3} z_{r} d_{2}=-e_{2}^{2}+e_{3} \beta_{0} z_{e}+e_{3} z_{r}\left(d_{2}-\beta(t)\right)=$ $\left(-e_{2}^{2}-\beta_{0} \cdot e_{3}^{2}\right)<0 \forall \beta_{0}>0$.

Then, Lyapunov's theorem about the asymptotic stability guarantees that $\lim _{t \rightarrow \infty} \vec{d}=\overrightarrow{0}$ and $\lim _{t \rightarrow \infty} \vec{e}=\overrightarrow{0}$; then $\left(x_{r}, y_{r}, z_{r}\right) \rightarrow\left(x_{e}, y_{e}, z_{e}\right)$ and $(\rho(t), \beta(t)) \rightarrow\left(\rho_{0}, \beta_{0}\right)$ if $t \rightarrow$ $\infty$.

Thus, any intruder system may synchronize the temporal evolution of the transmitter, deduct the values of the parameters and, in conclusion, break the cryptosystem. For that, the same synchronization signal employed in the receiver is enough.

In conclusion, Lorenz-based cryptosystems may be broken in a pretty easy way due to the low degree of complexity of that dynamics. Moreover, Orúe et al. [2] could prove how the Lorenz system is easily deciphered. Therefore, new more complex dynamics are necessary in order to build secure cryptosystems.

\section{A New Hyperchaotic Lorenz-Based System}

In order to get complex hyperchaos, we have considered the Lorenz system (1) and we have added a nonlinear controller $w$ to the first dynamical equation, including a differential nonlinear evolution law for $w$ as a new fourth component. We have sought to strengthen the rate of expansion and to reduce the rate of contraction of this dissipative system as explained below. From this procedure, we obtain the following new system:

$$
\begin{aligned}
& \dot{x}=d(y-x)+2 w, \\
& \dot{y}=5 x+c y-4 x z,
\end{aligned}
$$




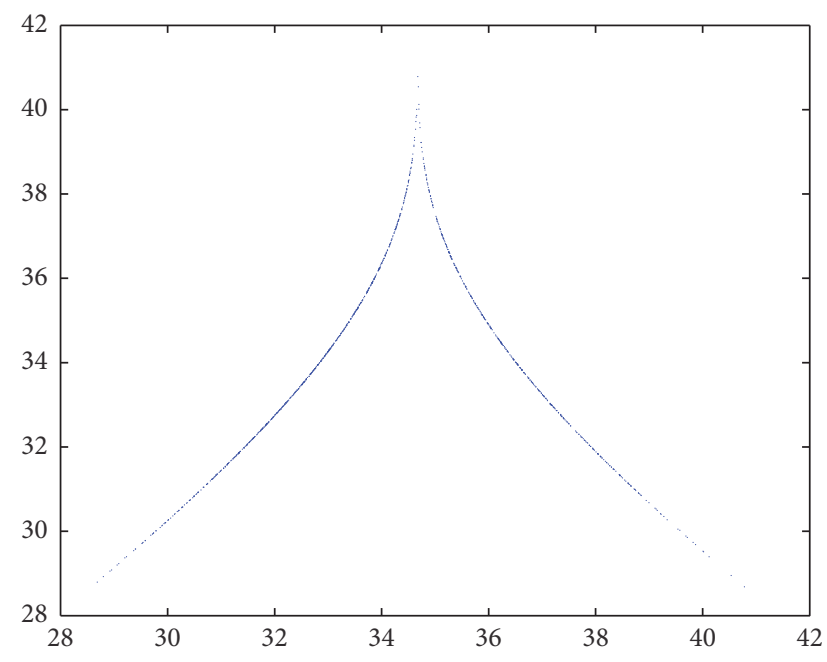

(a)

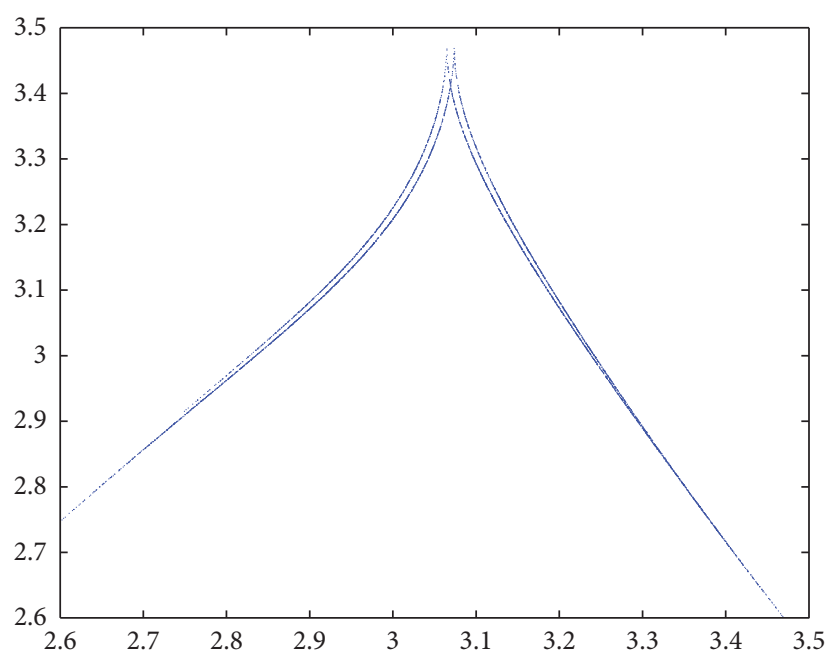

(b)

FiguRE 2: First return map for (a) Lorenz system $\{\sigma=10, \rho=178$, and $\beta=8 / 3\}$ and (b) proposed system $\{a=20, b=2, c=3$, and $d=10\}$.

$$
\begin{aligned}
& \dot{z}=x y-3 z, \\
& \dot{w}=-b w-a(x-y),
\end{aligned}
$$

where $b, c$, and $d$ are real positive parameters.

First of all, it must be noted that the proposed system still preserves the symmetry about $Z$-axis of the Lorenz dynamics. Nevertheless, as can be seen in Figure 2, this symmetry is not preserved in the first return map. One of the main discoveries of Lorenz in the system he investigated was the similarity of the first return map for chaotic trajectories to the tent map [21]. The tent map is the most basic paradigm of discrete chaos, being mainly characterized for its symmetry. As can be seen in Figure 2(b), in the proposed system (for chaotic trajectories), the first return map preserves some aspects of the Lorenz dynamics, but the resulting structure is not symmetric, as the cusp is splitted. This fact shows a first sign of the degree of complexity reached.

On the other hand, the proposed system presents three different fixed points (16) that maintain the symmetry about $Z$-axis, inherited from the Lorenz system:

$$
\begin{gathered}
P 0(0,0,0,0), \\
P 1\left(\sqrt{\frac{3}{4}(5+c)}, \sqrt{\frac{3}{4}(5+c)}, \frac{5+c}{4}, 0\right), \\
P 2\left(-\sqrt{\frac{3}{4}(5+c)},-\sqrt{\frac{3}{4}(5+c)}, \frac{5+c}{4}, 0\right) .
\end{gathered}
$$

As can be seen, $P 0$ (the coordinate origin) does not depend on any parameter, and $P 1$ and $P 2$ depend only on the parameter $c$. Then, as parameter $c$ is always positive, the coordinates of $P 0, P 1$, and $P 2$ are always real and different. The three points, which lie on the hyperplane $w=0$, are getting closer as parameter $c$ decreases (without ever colliding). Therefore, the system exhibits no bifurcations involving collisions or splits of the fixed points such as the pitchfork bifurcation.

Other important requirements for the novel system are as follows. (i) We maintain the two nonlinear terms of the Lorenz system but we introduce one new divergence in the second equation of system (15) with the aid of the parameter c. (ii) The system has dissipative structure and therefore the four dynamics parameters cannot be fixed at any particular value independently (17). This is a necessary condition for dissipative chaos that has to be fulfilled. The volume occupied by the system in the phase space should decrease as time goes on. In dynamical systems theory, this is verified by checking that the rate of the occupied volume per volume unit is negative (i.e., the rate of volume contraction):

$$
\frac{1}{V}\left(\frac{d V(t)}{d t}\right)<0 .
$$

Expressing the dynamics as a matrix, we obtain the following:

$$
\begin{aligned}
\left(\begin{array}{c}
\dot{x} \\
\dot{y} \\
\dot{z} \\
\dot{w}
\end{array}\right) & =\vec{F}=\left(\begin{array}{c}
d(y-x)+2 w \\
5 x+c y-4 x z \\
x y-3 z \\
-b w-a(x-y)
\end{array}\right), \\
\frac{1}{V}\left(\frac{d V(t)}{d t}\right) & =\operatorname{div}(\vec{F})=\left(\frac{\partial \dot{x}}{\partial x}+\frac{\partial \dot{y}}{\partial y}+\frac{\partial \dot{z}}{\partial z}+\frac{\partial \dot{w}}{\partial w}\right) \\
& =c-(d+3+b) .
\end{aligned}
$$

So, the dynamics parameters must verify the following condition:

$$
d>c-(b+3) \text {. }
$$

As can be seen, the described dynamics depends on four parameters. However, in order to analyze the new system, it 
TABLE 1: Local stability study.

\begin{tabular}{|c|c|c|c|c|c|}
\hline Analysis & Parameters & Eigenvalues $P 0$ & Interpretation & Eigenvalues $P 1, P 2$ & Interpretation \\
\hline \multirow{5}{*}{$\begin{array}{l}\text { System evolution } \\
\text { when varying the } \\
\text { parameter } a \text { for } \\
d=10\end{array}$} & $0<a<3$ & $r_{1}^{+}, r_{2}^{-}, r_{3}^{-}, r_{4}^{-}$ & $\begin{array}{l}\text { Saddle point } \\
\text { order } 1\end{array}$ & $c_{1}^{+}, c_{2}^{+}, r_{3}^{-}, r_{4}^{-}$ & $\begin{array}{c}\text { Saddle spiral } \\
\text { order } 2\end{array}$ \\
\hline & $3<a<15$ & $r_{1}^{+}, r_{2}^{-}, r_{3}^{-}, r_{4}^{-}$ & $\begin{array}{l}\text { Saddle point } \\
\text { order } 1\end{array}$ & $c_{1}^{+}, c_{2}^{+}, c_{3}^{-}, c_{4}^{-}$ & $\begin{array}{c}\text { Saddle spiral } \\
\text { order } 2\end{array}$ \\
\hline & $15<a<25$ & $r_{1}^{+}, r_{2}^{-}, c_{3}^{-}, c_{4}^{-}$ & $\begin{array}{l}\text { Saddle spiral } \\
\quad \text { order } 1\end{array}$ & $c_{1}^{+}, c_{2}^{+}, c_{3}^{-}, c_{4}^{-}$ & $\begin{array}{c}\text { Saddle spiral } \\
\text { order } 2\end{array}$ \\
\hline & $a=25$ & \multicolumn{4}{|c|}{ Hopf bifurcation } \\
\hline & $a>25$ & $r_{1}^{+}, r_{2}^{-}, c_{3}^{-}, c_{4}^{-}$ & $\begin{array}{c}\text { Saddle spiral } \\
\text { order } 1\end{array}$ & $c_{1}^{-}, c_{2}^{-}, c_{3}^{-}, c_{4}^{-}$ & Spiral node \\
\hline $\begin{array}{l}\text { System evolution } \\
\text { when varying the } \\
\text { parameter } a \text { for } d=2\end{array}$ & $\forall a$ & $r_{1}^{+}, r_{2}^{-}, c_{3}^{-}, c_{4}^{-}$ & $\begin{array}{l}\text { Saddle spiral } \\
\text { order } 1\end{array}$ & $c_{1}^{+}, c_{2}^{+}, c_{3}^{-}, c_{4}^{-}$ & $\begin{array}{l}\text { Saddle spiral } \\
\text { order } 2\end{array}$ \\
\hline \multirow{4}{*}{$\begin{array}{l}\text { System evolution } \\
\text { when varying the } \\
\text { parameter } d \text { for } \\
a=20\end{array}$} & $0<d<11$ & $r_{1}^{+}, r_{2}^{-}, c_{3}^{-}, c_{4}^{-}$ & $\begin{array}{l}\text { Saddle spiral } \\
\text { order } 1\end{array}$ & $c_{1}^{+}, c_{2}^{+}, c_{3}^{-}, c_{4}^{-}$ & $\begin{array}{l}\text { Saddle spiral } \\
\text { order } 2\end{array}$ \\
\hline & $d=11$ & \multicolumn{4}{|c|}{ Hopf bifurcation } \\
\hline & $11<d<12$ & $r_{1}^{+}, r_{2}^{-}, c_{3}^{-}, c_{4}^{-}$ & $\begin{array}{l}\text { Saddle spiral } \\
\text { order } 1\end{array}$ & $r_{1}^{-}, r_{2}^{-}, c_{3}^{-}, c_{4}^{-}$ & Spiral node \\
\hline & $d>12$ & $r_{1}^{+}, r_{2}^{-}, r_{3}^{-}, r_{4}^{-}$ & $\begin{array}{l}\text { Saddle point } \\
\text { order } 1\end{array}$ & $r_{1}^{-}, r_{2}^{-}, c_{3}^{-}, c_{4}^{-}$ & Spiral node \\
\hline
\end{tabular}

is important to choose a parameter configuration which not only maintains the system's complexity but also simplifies its analysis. Therefore, we are taking fixed values for some system parameters. We fix the degree of divergence which controls the parameter $c$ to $c=3$ and finally the parameter $b$, which controls the convergence, is set to $b=2$. If parameters $b$ and $c$ are fixed, the parameter $d$ obeys $>-2$.

For $d<-2$, the divergence is positive and the system tends to be not steady and diverges. When $d$ is near zero, the system tends to converge slowly and generates hyperchaotic structures. In particular, when $d=2$ and $a=38$, we obtain a high value of the maximum Lyapunov exponent of approximately $\lambda_{\max }=12$ and a Kaplan-Yorke dimension close to $D_{\mathrm{KY}}=4$. In contrast, when $d>0$, if $d$ becomes larger, system (15) shrinks strongly and will tend to be steady towards one of its equilibrium points. Thus, the parameter $a$ can be varied independently without change in the general behavior, which is determined essentially by the parameter $d$. Then, by the introduction of $a$ and, especially, $d$ as control parameters, we allow exploring the regular, chaotic, and hyperchaotic topologies of the system.

According to these arguments and in order to study the maximum number of different representative structures and topologies, three different analyses are going to be performed:

(1) System evolution when varying the parameter $a$ for $d=10$

(2) System evolution when varying the parameter $a$ for $d=2$

(3) System evolution when varying the parameter $d$ for $a=20$

For all the three analyses described above, the eigenvalues associated with the matrix of the linearized system around the equilibrium points have no analytical expression depending on the dynamics parameters. Thus, to obtain their values and study the stability of the fixed points, possible bifurcations, and so forth, we made a numerical calculation. We take advantage in considering the symmetry of the system and we know, in general, that the linearized systems around the points $P 1$ and $P 2$ have the same eigenvalues, so their stability and behavior are identical. Table 1 studies the behavior of the fixed points for the three situations identified above.

From the analysis of the results shown in Table 1, two conclusions about the global behavior of the system may be deduced:

(1) Taking $d=10$, the evolution of the system is analyzed when varying the parameter $a$. Then a Hopf bifurcation appears at $a=25$. At this point, the exterior fixed points change their behavior from instable to asymptotically stable. For values lower than but close to $a=25$, the system admits (as we see in Section 4) two coexisting limit cycles which tend to be smaller when getting closer to $a=25$ until they collapse over the fixed points. Therefore, at $\{a=25, b=2, c=3$, and $d=10\}$ the system presents a supercritical Hopf bifurcation.

(2) On the other hand, with $a=20$, the evolution of the system is analyzed when varying the parameter $d$. Then another supercritical Hopf bifurcation appears at $d=11$. The system behavior is identical to what was described previously for the value $a=25$.

Various methods to determine the supercritical character of Hopf bifurcations have been described in the research literature. In particular, a Hopf bifurcation is considered subcritical if the maximum Lyapunov exponent in the bifurcation point is positive and supercritical in the opposite case. 
TABLE 2: Lyapunov spectrum at the bifurcation points.

\begin{tabular}{lcccc}
\hline Case & \multicolumn{3}{c}{ Lyapunov spectrum } \\
\hline$\{a=25, b=2, c=3$, & $\left(\begin{array}{lllll}-0.0419 & -0.0503 & -5.9441 & -5.9613\end{array}\right)$ \\
$\begin{array}{l}\text { and } d=10\} \\
\{a=20, b=2, c=3,\end{array}$ & $\left(\begin{array}{lllll}-0.0282 & -0.0341 & -6.4436 & -6.4914\end{array}\right)$ \\
and $d=11\}$
\end{tabular}

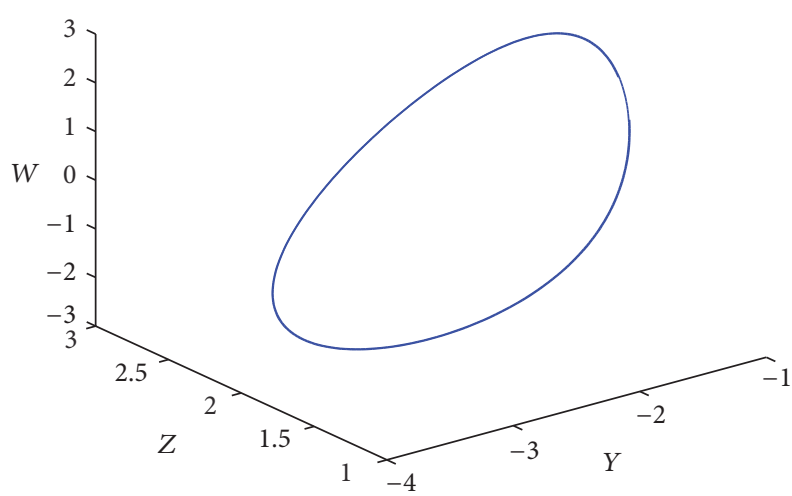

Figure 3: Projection over the subspace $Y Z W$ of the limit cycle of new system, working in regular region $(a=25$ and $d=10)$.

Numerical algorithms allow determining the Lyapunov spectrum of the two cases of interest. Results are shown in Table 2. As can be seen, in both cases, the maximum Lyapunov exponent is negative, so, in fact, bifurcations are supercritical.

In the proposed system, the complexity of the trajectories tends to increase for small values of parameter $d$, since the system is increasingly less convergent, as we will show later. Thus, hyperchaotic and more complex structures are located when analyzing the system evolution along the parameter $a$ for $d=2$. In this range of low values of the parameter $d$, strong exponential growing (accompanied by strong contractions, since the system is dissipative) and instabilities appear, so we will do a careful study about the obtained results.

\section{Regular Structures}

Regular structures may be found in any of the three studies proposed. In particular, two different regular types of trajectories are generated by the proposed system. On one hand, common limit cycles are generated in the neighborhoods of the Hopf bifurcations identified in the previous section. On the other hand, more complex cycles are generated for different values of the parameter $a$ when considering the value $d=10$.

In the next subsections, both cases are studied.

4.1. Limit Cycles and Hopf Bifurcation. Limit cycles (Figure 3) are mainly located in two ranges (both in the neighborhoods of the Hopf bifurcations): $a \in(22.5,25)$ for $d=10$ and $d \epsilon$ $(10.5,11)$ for $a=20$.

In both cases, two different limit cycles coexist at the same time (as can be seen in Figure 4), with each one developing around of one of the exterior fixed points $P 1$ and $P 2$.

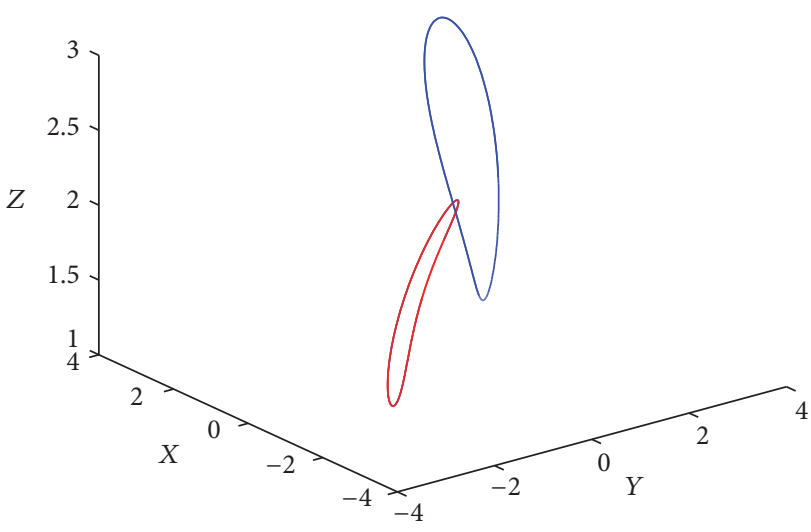

(a)

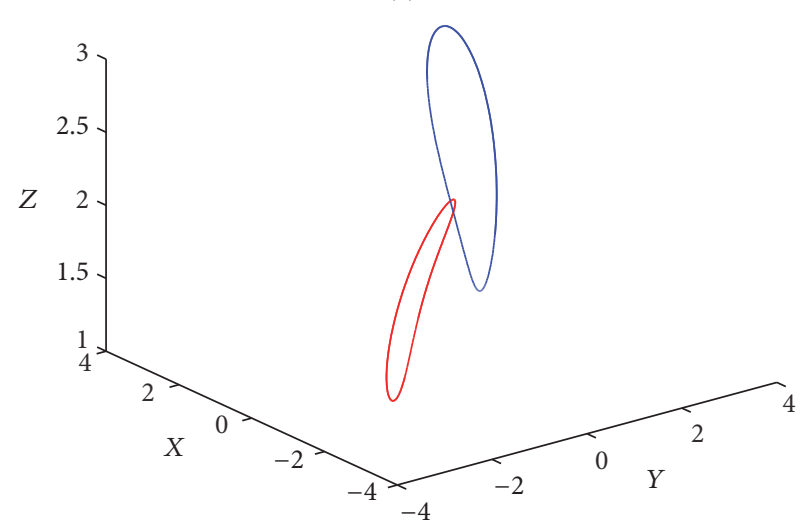

(b)

FIgURE 4: Coexisting limit cycles in the proposed system. (a) $\{a=$ $24, b=2, c=3$, and $d=10\}$ and (b) $\{a=20, b=2, c=3$, and $d=10.55\}$. Initial conditions: $(1,1,1,1)$ and $(-2,-2,-2,-2)$.

This coexistence is maintained while the system remains in the regular region and disappears when chaotic trajectories appear (as these trajectories are developed around both fixed points). This fact is clearly shown in Figure 5, where a bifurcation diagram for two different initial conditions is represented. As can be seen, in the regular region, both diagrams (one represented in red color and the other in green color) evolve in parallel and independently. Nevertheless, when chaotic structures appear, both diagrams overlap.

Finally, the existence of a Hopf bifurcation must be proven. In Figure 6, a composition of the evolution of the limit cycles when varying the control parameters is provided. As can be seen, limit cycles reduce their size when getting closer to the bifurcation point, until they collapse over the fixed points. This behavior is, in fact, corresponding to a supercritical Hopf bifurcation.

4.2. Other Regular Attractors and the Corresponding Bifurcation Diagram. If the system flow evolution when varying the parameter $a$ for $d=2$ is considered, more complex structures are found (see Figure 7). A rich structure of regular trajectories appears within certain windows and miniature windows within the larger windows of parameter $a$. In this figure (Figure 7), two bifurcation diagrams are shown, representing 


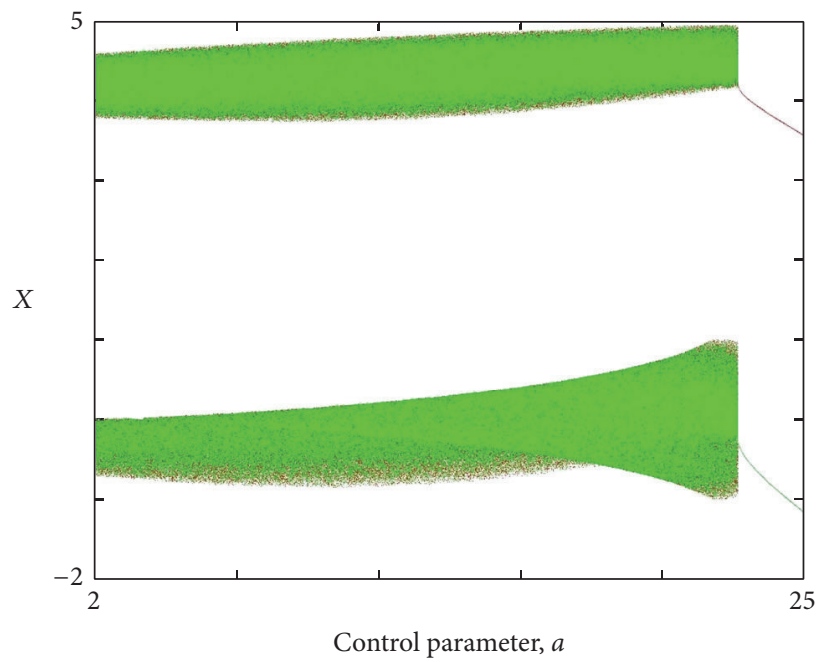

FiguRE 5: Bifurcation diagram using $a$ as control parameter for two different initial conditions. In red color $(1,1,1,1)$ and in green color $(-2,-2,-2,-2)$. Parameters: $\{b=2, c=3$, and $d=10\}$.

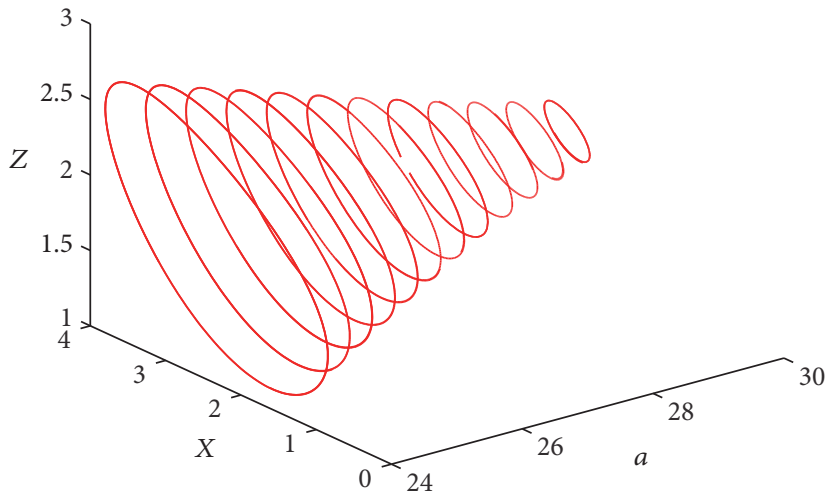

Figure 6: The evolution of the limit cycles with the parameter $a$ in the neighborhood of Hopf bifurcation. Initial conditions: $(1,1,1,1)$. Parameters: $\{b=2, c=3$, and $d=10\}$.

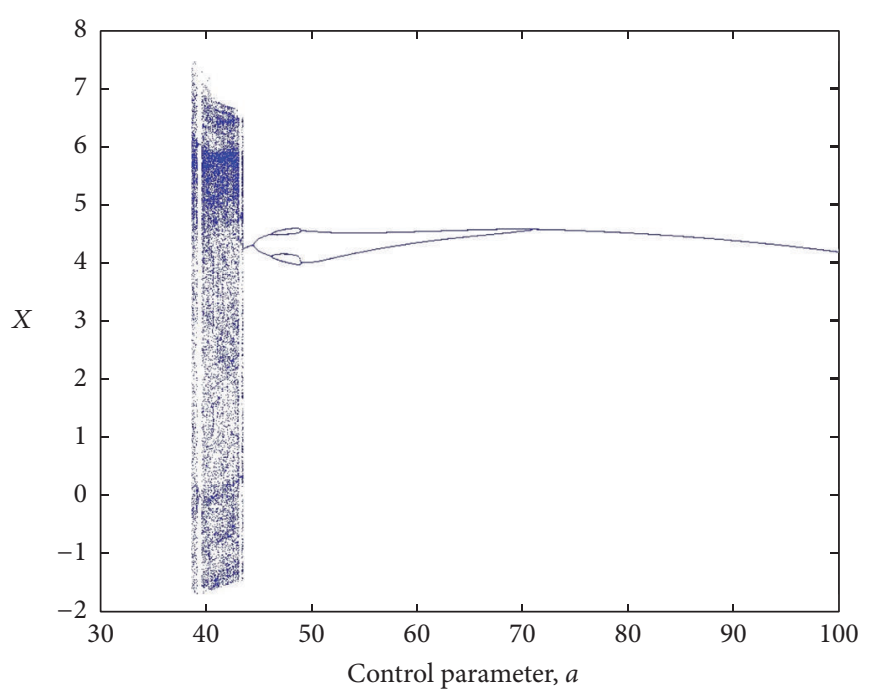

(a)

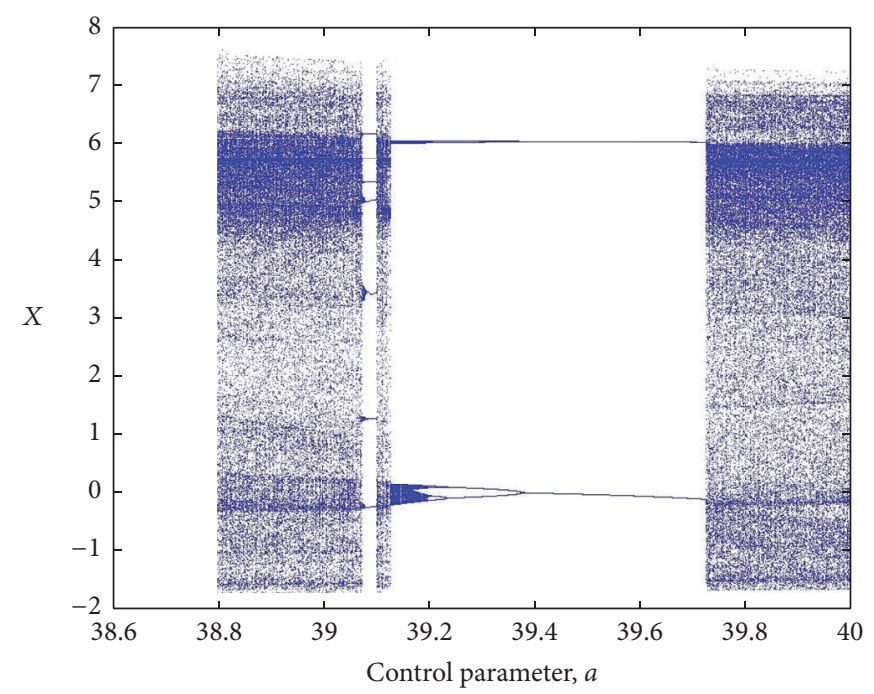

(b)

Figure 7: Bifurcation diagram using $a$ as control parameter. Parameters: $\{b=2, c=3$, and $d=2\}$. 


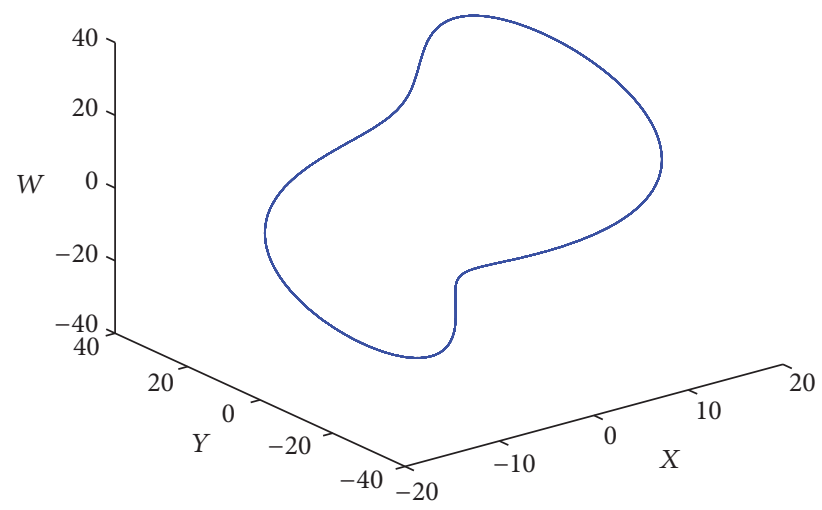

(a)

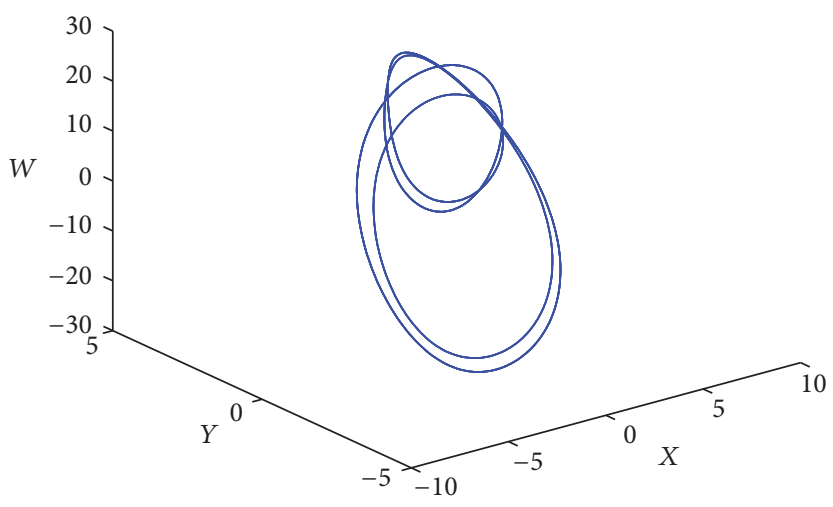

(b)

FIgure 8: Complex limit cycle for (a) $\{a=10$ and $d=3\}$ and (b) $\{a=50$ and $d=2\}$. Projection over the subspace XYW.

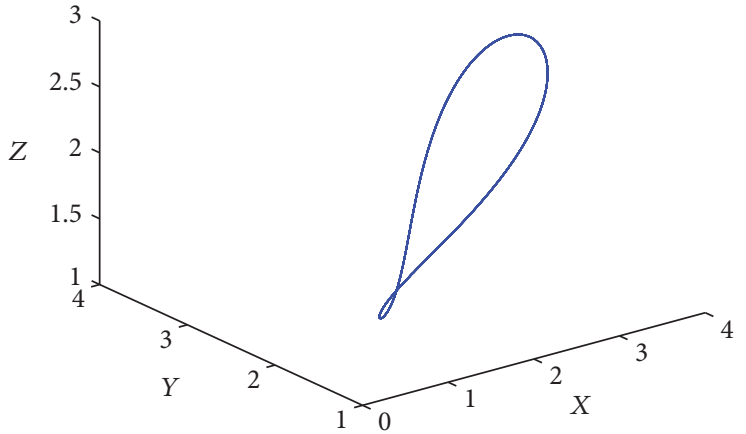

(a)

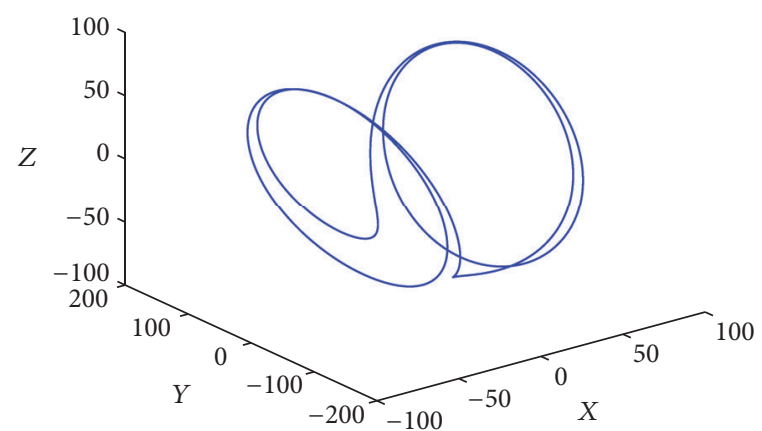

(c)

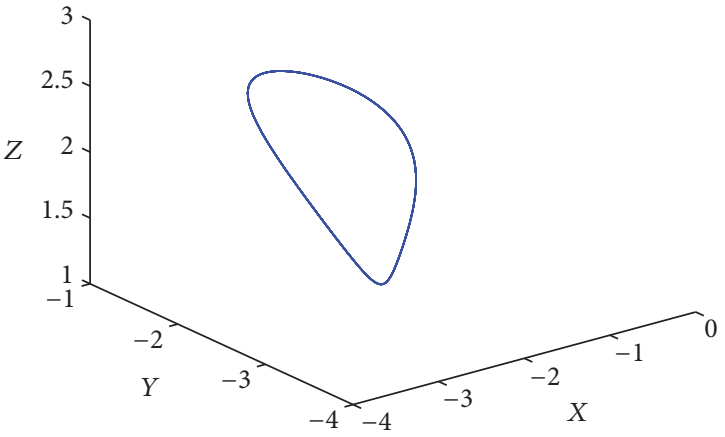

(b)

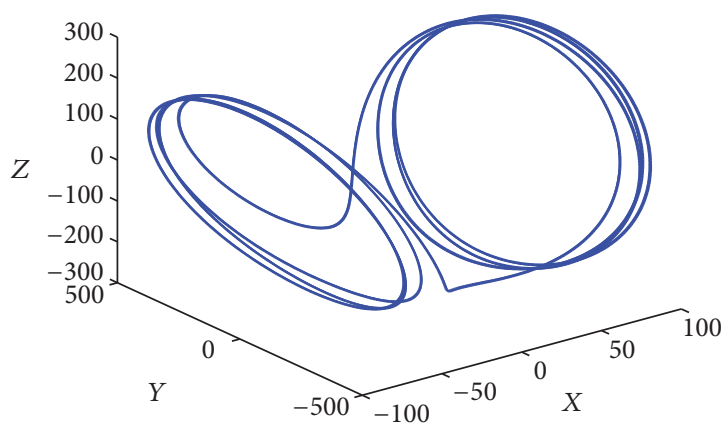

(d)

Figure 9: Coexisting limit cycles for $\{a=40$ and $d=7\}$. Initial conditions: (a) $(150,2,1,45),(\mathrm{b})(0.1,0.1,0.1,0.1),(\mathrm{c})(100,50,50,100)$, and (d) $(200,300,200,350)$.

different regular and chaotic regions in various ranges of the parameter $a$ for $d=2$.

Several and interesting bifurcations may be identified in these diagrams. For example, at $a=70$, a period doubling bifurcation is exhibited. Additionally, around $a=50$, another period doubling cascade gets stopped and reversed. Moreover, in the range $a \in(39.1,39.7)$, a period doubling cascade, a chaotic window, and a period doubling again end at $a=39.7$ in chaos. As can be seen, at $a=39.7$, the chaos appears abruptly by means of a tangent bifurcation. At this bifurcation parameter value, an underlying saddle point and an underlying stable node collide and annihilate one another. As a result, an orbit that has periods of chaos interspersed with periods of regular oscillation is generated
[22]. The same ideas shown before allow identifying another tangent bifurcation at $a=43$.

In some of the shown areas, limit cycles appear (e.g., in the range $a \in(70,100)$; see Figure 8(a)). However, other interesting regular structures are also generated, as can be seen in Figure 8(b).

On the other hand, although the couple $\{a=40$ and $d=7\}$ does not belong to any of the proposed analyses, the observed structures deserve to be commented on. In fact, in this range, the complexity of the system is enormous, while up to four different hidden limit cycles [23] can be generated by the dynamics for $\{a=40$ and $d=7\}$ modifying the initial conditions (see Figure 9). Some of these cycles are regular limit cycles, but others exhibit a great complexity. 


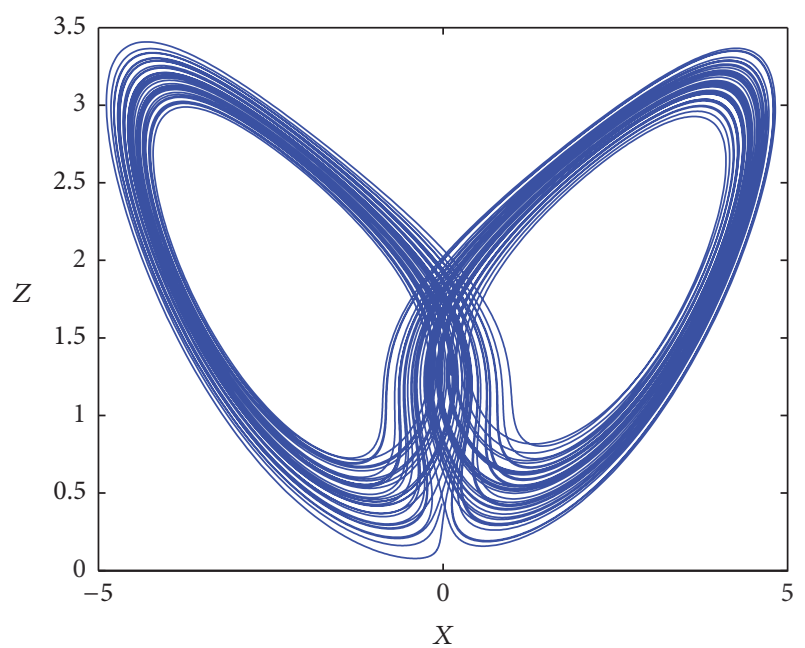

FIGURE 10: Lorenz-like chaotic attractor in the proposed system $\{a=$ 20 and $d=10\}$.

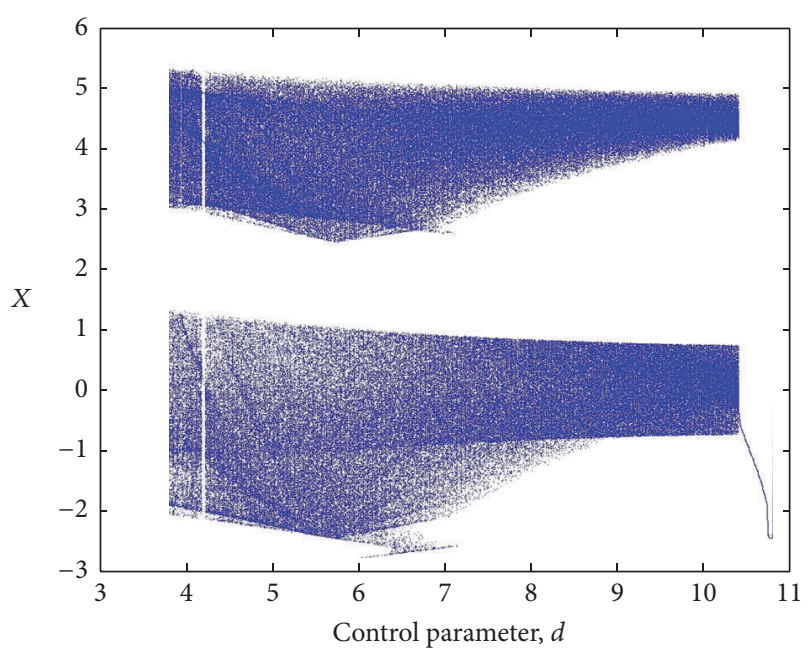

Figure 11: Bifurcation diagram using $d$ as control parameter. Parameters: $\{a=20, b=2$, and $c=3\}$.

\section{Chaotic Structures}

In the proposed system, the weakly chaotic structures maintain certain similarity with the classic Lorenz attractor (see Figure 10). In this figure, a pretty similar attractor to Lorenz's butterfly is shown and, as it was seen in Figure 2(b), the first return map associated with this topology is also similar to that obtained in the Lorenz dynamics (apart from the changes in the symmetry). This chaotic region is well appreciated in the diagram of Figure 11, where parameter $a$ is fixed to $a=20$ and the parameter $d$ is variable. We can appreciate that in the region $d \in(3.8,10.4)$ the dynamics is chaotic, and when $d>10.4$ it starts the regular regime that finally converges to $P 0$ fixed point by means of a Hopf bifurcation.

Other interesting structures in the phase space, together with the associated first return map, can be seen in Figure 12. The topology generated for $\{a=20$ and $d=7\}$ maintains a great similarity to Lorenz's butterfly, but in the first return
TABLE 3: Lyapunov exponents study.

\begin{tabular}{lcc}
\hline Topology & Lyapunov exponents & Kaplan-Yorke dimension \\
\hline$a=20 ; d=7$ & $(0.62,0,-4.7,-4.91)$ & 2.12 \\
$a=32 ; d=5$ & $(0.43,0,-3.2,-4.1)$ & 2.13 \\
$a=40 ; d=2$ & $(1.11,0,-2.3,-2.7)$ & 2.47 \\
\hline
\end{tabular}

map there is a double cusp more complex than the Lorenz return map for $Z$-component. The beginning of the symmetry breaking can also be seen. Similar topologies may be found using the bifurcation diagram provided in Figure 11. For $\{a=32$ and $d=5\}$, the variations are really evident. The first return map still contains some information about the Lorenz dynamics but has only a slight influence. At this point, complexity has started growing up.

As we have said previously and as can be seen in the bifurcation diagram of Figure 7, the most complex structures are located in the system evolution when varying the parameter $a$ for $d=2$. In these ranges, highly complex chaotic structures and hyperchaotic trajectories may be located. In particular, for the couple of parameters $\{a=40$ and $d=2\}$ a high complexity chaotic solution is generated. As can be seen, the trajectory in the phase space is much more complex than that obtained for the Lorenz dynamics. Moreover, the first return map is an almost-random cloud of points which does not maintain any symmetry or regular structure as in the previous cases.

Table 3 provides a study about the Lyapunov exponents and the Kaplan-Yorke dimension of the structures shown in Figure 12, which proves the increase of the complexity as the simpler Lorenz dynamics disappears.

In particular, Figure 12(c) shows a high complex chaotic structure, which presents a Kaplan-Yorke dimension, $D_{\mathrm{KY}}=$ 2.47. In the range $a \in(38,40)$, various additional complex trajectories may be found, including hyperchaotic solutions coexisting with high complexity chaotic trajectories. However, in the range $a \in(40,43)$, the proposed system behaves chaotically and various strange attractors with a low similarity to Lorenz butterfly are found (see Figure 13).

As can be seen and as was said in Section 3, the increase of the complexity is due to the explicit control of rates of convergence and divergence by means of the system parameters. As a final result and with varying the parameters $a$ and $d$, the hyperchaotic behavior appears. Next section is dedicated to these trajectories.

\section{Hyperchaotic and Other Complex Structures}

In this section, different complex chaotic structures are located and analyzed. As we will see below, in the proposed system, the strengthening of the expansion dimensions has motivated the appearance of highly complex chaotic trajectories, sometimes coexisting with hyperchaotic ones. Other complex structures (such as the chaotic-transient solutions) and unbounded solutions, when the parameter $d$ is small (in absolute value), also appear.

These structures are reviewed in the following subsections. 

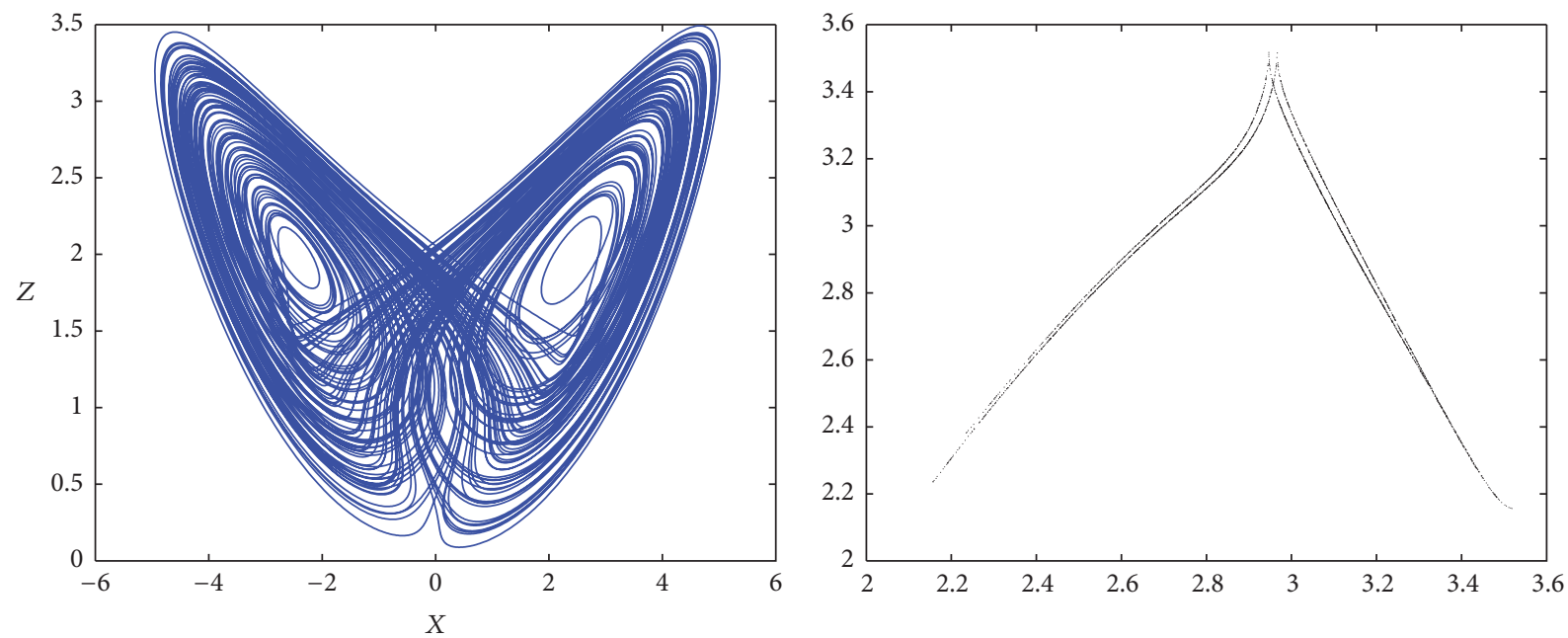

(a)
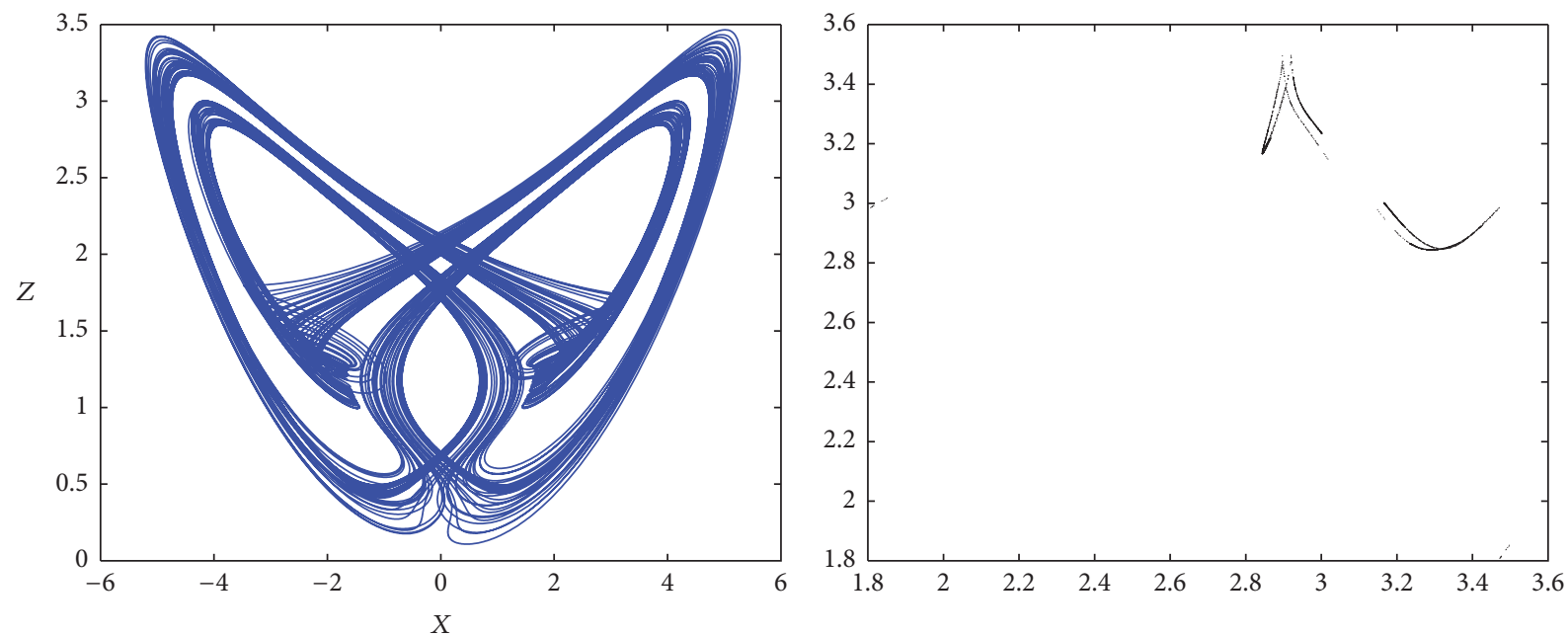

(b)
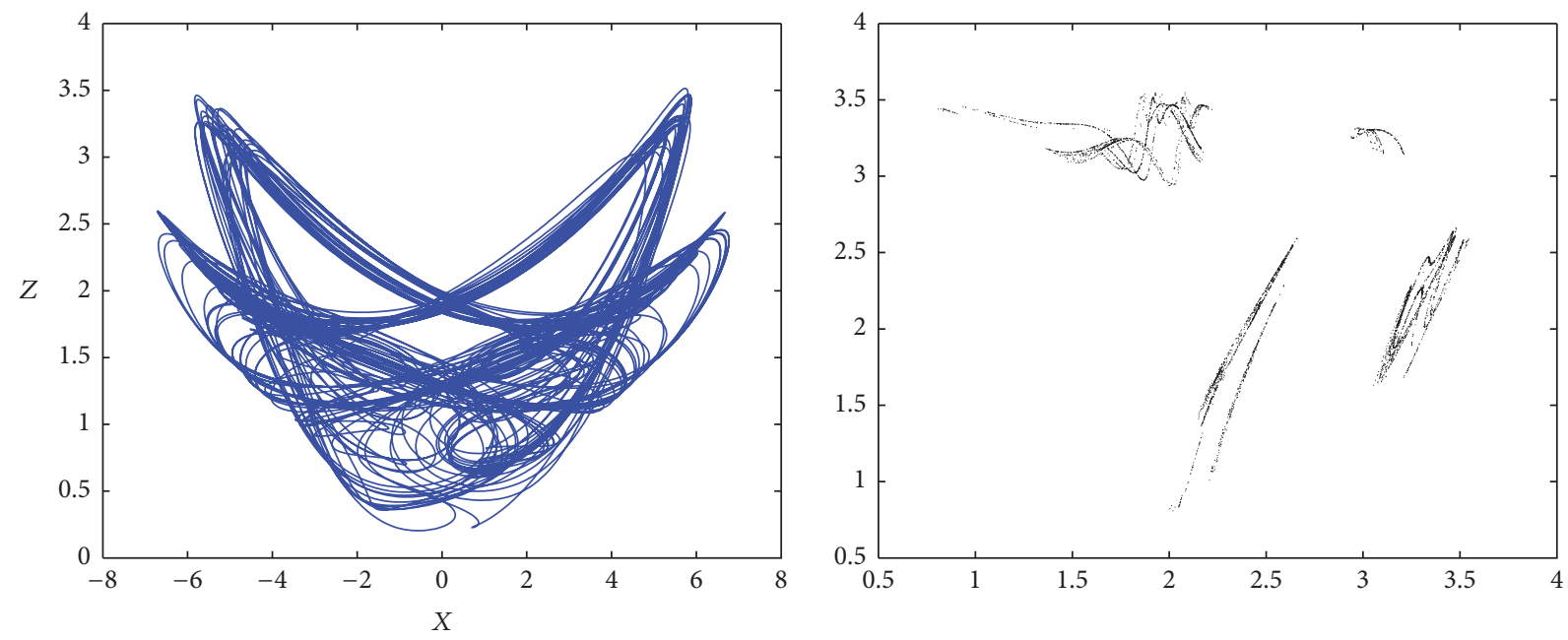

(c)

Figure 12: Chaotic attractors of the $X Z$ components generated by the proposed system and first return maps obtained from $Z$-component. Initial conditions: $(0.1,0.1,0.1,0.1)$. Parameters: (a) $\{a=20$ and $d=7\}$, (b) $\{a=32$ and $d=5\}$, and (c) $\{a=40$ and $d=2\}$. 


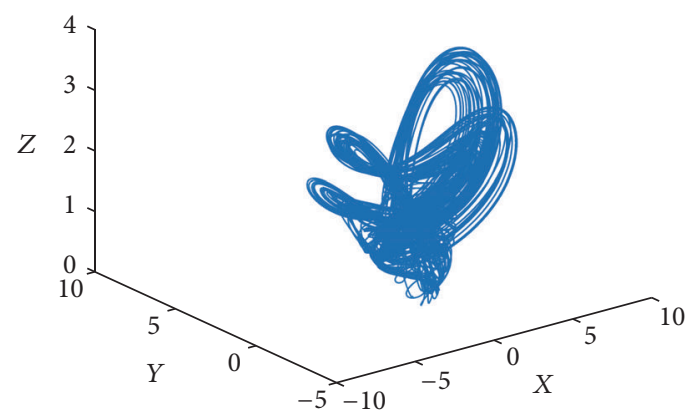

(a)

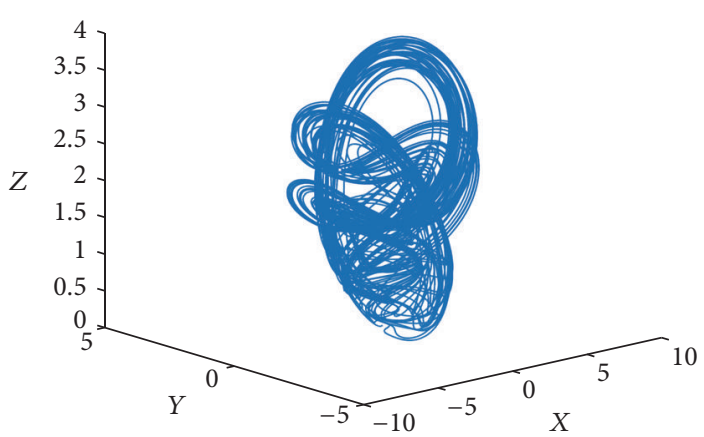

(b)

FIGURE 13: Chaotic tridimensional attractors generated by the proposed system. Initial conditions: $(0.1,0.1,0.1,0.1)$. Parameters: (a) $\{a=40$ and $d=2\}$ and (b) $\{a=43$ and $d=2\}$.

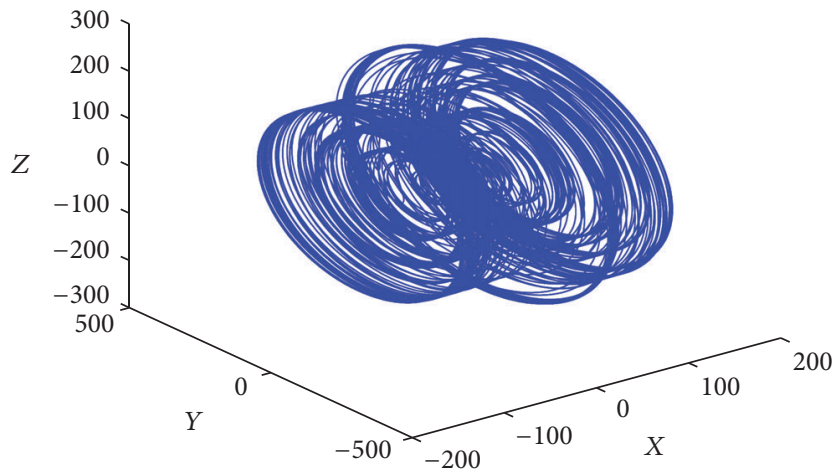

FIGURe 14: Projection over the subspace $X Y Z$ of the chaotictransient solution.

6.1. Chaotic-Transient Solutions. As we have commented in the previous subsection, hyperchaotic and unbounded solutions coexist in most of the cases. However, another type of solutions which may be confused with hyperchaotic structures can exist. In some cases, the proposed system generates trajectories which seem to be hyperchaotic at first. However, as time elapses, the components enter in a coherent state which does not correspond with hyperchaos. This situation, for example, appears for the couple of parameters $\{a=10$ and $d=2\}$.

Figure 14 shows a projection of the cited trajectory over the subspace $X Y Z$. As can be seen, a cylindrical topology appears, indicating an almost-regular behavior in some of the components. At the first moments, this structure generates a Kaplan-Yorke dimension around $D_{\mathrm{KY}}=3.4$, but, as time elapses, the system components reach a coherent state and complexity goes down rapidly.

In fact, $Z$-component and $Y$-component (as well as $X$ component and $W$-component) evolve together with regular oscillations from $t>30$ dimensionless units (see Figure 15). In conclusion, these topologies present a chaotic structure in a transitory way, but, as time elapses, they become more regular and lose complexity.

Figure 16 shows first two return maps, where the chaotic behavior can be seen on one hand and, on the other hand,
TABLE 4: Lyapunov exponents study for the hyperchaotic structure.

\begin{tabular}{lcc}
\hline Exponent & Value & Kaplan-Yorke dimension \\
\hline$\lambda_{1}$ & 12.37 & \\
$\lambda_{2}$ & 0 & 3.83 \\
$\lambda_{3}$ & -0.036 & \\
$\lambda_{4}$ & -14.71 & \\
\hline
\end{tabular}

the regular behavior can be seen. As can be seen, the regular regime is clearly distinguished by the cloud of points over $y=x$ straight line which is especially dominant in the return map of $Y$-component (Figure 16(b)). On the other hand, the behavior of the chaos is characterized, in both figures, from a dense cloud of points outside of the straight line. In addition to these arguments, in Figure 15, it can be seen that the regular trajectory evolution at about $t>40$ is dominant, indicating that, at the end, this regular trajectory is the cause of the loss of complexity.

6.2. Hyperchaotic Solutions. Although, probably, different hyperchaotic structures could be generated by the proposed system using different values of the control parameters, in this section, we will focus the study on the behavior in the range $a \in(38,38.5)$ taking the value $d=2$.

The first hyperchaotic structure may be found at $a=$ 38 , although in the range $a \in(38,38.5)$ similar result could be obtained. Figure 17 shows the four different threedimensional projections of the four-dimensional specified attractor.

A basic proof of the presence of hyperchaos is obtained by means of the Lyapunov spectrum. Table 4 shows its values. As can be seen, in this system, like the generalized Chen system [11], only one exponent is positive and shows a high value. However, the third exponent is really close to zero (although it is negative).

Using the ordered Lyapunov spectrum $\left(\lambda_{1}>\lambda_{2}>\lambda_{3}>\right.$ $\lambda_{4}$ ) we can observe a great divergence in the direction of the first exponent together with a low contraction in the direction of the third exponent. This is the reason for the great complexity of the hyperchaotic solution being analyzed. The Kaplan-Yorke dimension $D_{\mathrm{KY}}$ clearly shows this high 

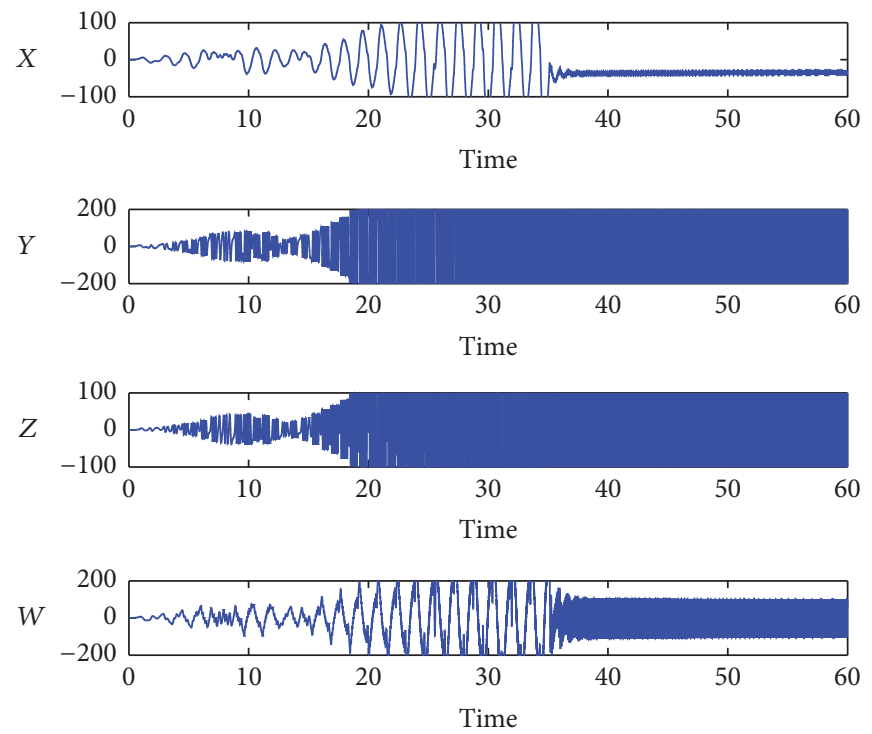

FIGURE 15: Temporal evolution in the chaotic-transient solutions.

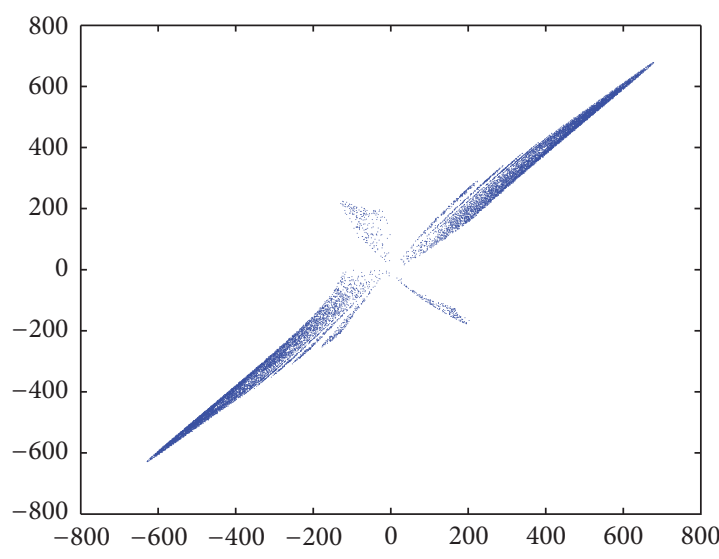

(a)

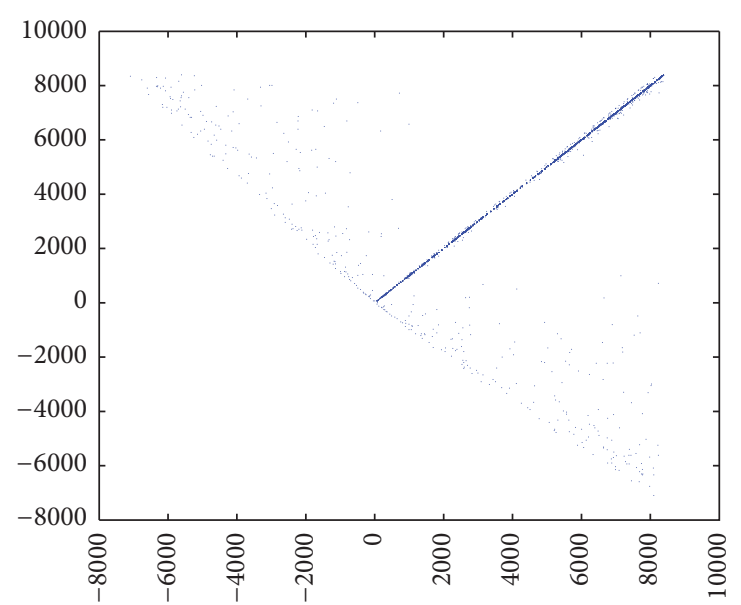

(b)

Figure 16: First return map for the chaotic-transient solution. (a) $X$-component; (b) $Y$-component.

complexity. It presents a value greater than three and close to four $\left(D_{\mathrm{KY}}=3.83\right)$.

Also, we can observe that $\lambda_{1}$ and $\lambda_{4}$, with opposite signs, compete with each other. Baier and Thomsen [24] performed an analysis of the complexity of the chaotic attractors in four dimensions and established the following:

(a) For $\lambda_{1}>0$ and $\left(\lambda_{1}+\lambda_{3}\right)<0$, there are ordinary chaos with $2<D_{\mathrm{KY}}<3$.

(b) For $\left(\lambda_{1}+\lambda_{2}\right)>0$ and $\left(\lambda_{1}+\lambda_{3}\right)>0$, there appears more complex chaos (hyperchaos) with $3<D_{\mathrm{KY}}<4$.

Our case belongs to the more complex chaotic behavior indicated in (b).

One of the problems when working with dynamical systems with a high level of complexity is the flow instability depending on the control parameters in the region of hyperchaos and transient chaos. This high level of complexity appears for low values of $d$ parameter $(d \leq 2)$ and with $a<37$, and it is very sensitive on integration errors. In fact, precise integration algorithms and Lyapunov spectrum calculation routines are necessary in order to obtain accurate data.

In the case of the result shown in Table 4, both routines, the integration one (with a Runge-Kutta four-order method and a fifth-order error control) and the Lyapunov spectrum one, have been implemented with a relative error between $10^{-7}$ and $10^{-8}$ and with a very long time propagation, so the convergence is reached, as can be seen in Figure 18. To obtain the Lyapunov spectrum, we use Wolf et al.'s algorithm [25] with a temporal step size between $10^{-3}$ and $10^{-4}$.

Finally, since the four-dimensional attractors cannot be represented in only one graphic, Poincaré sections are a traditional way of proving the complexity level and the presence of chaos. Although the Lyapunov exponents and the previous results prove the hyperchaotic behavior of the analyzed structure, Poincaré sections in Figure 19 demonstrate the existence of the hyperchaos in the system. These 


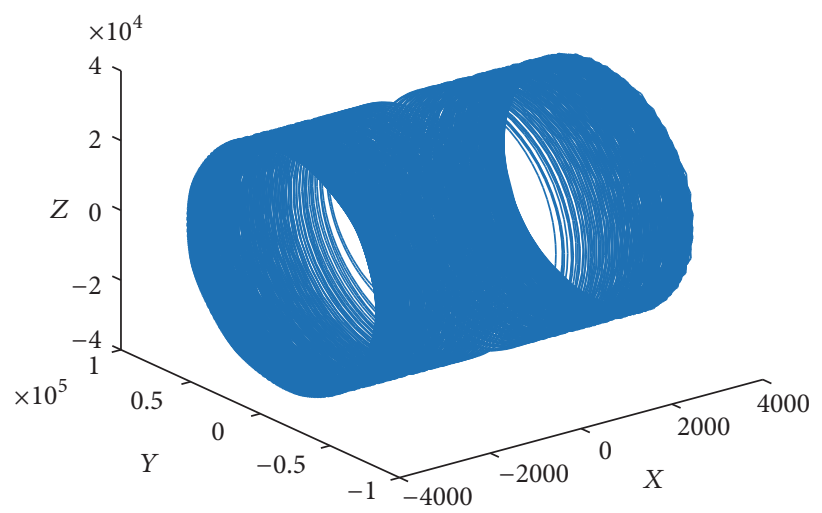

(a)

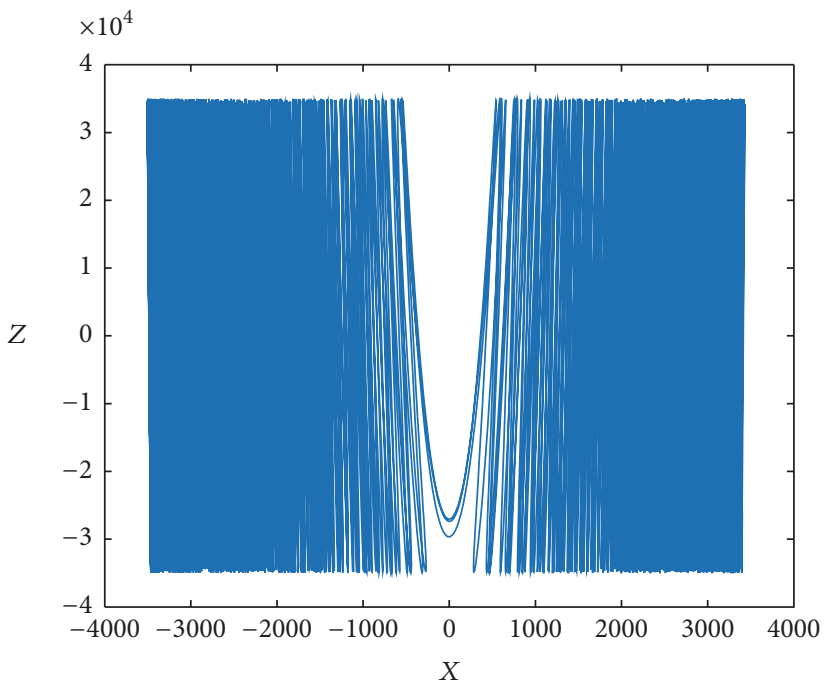

(c)

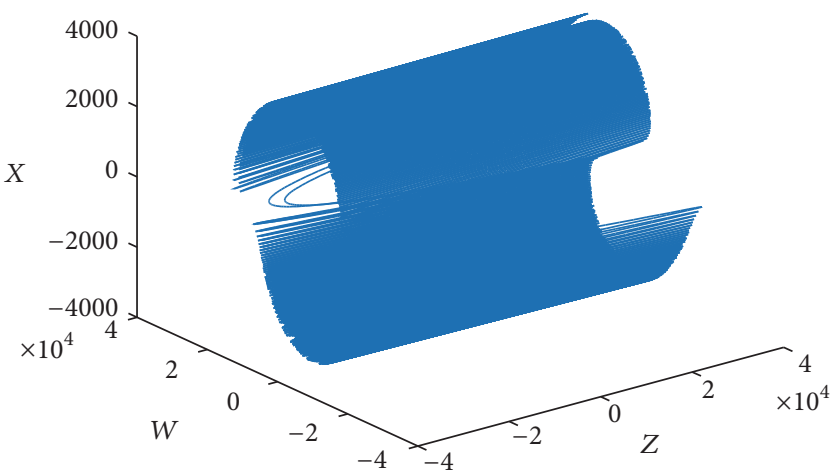

(b)

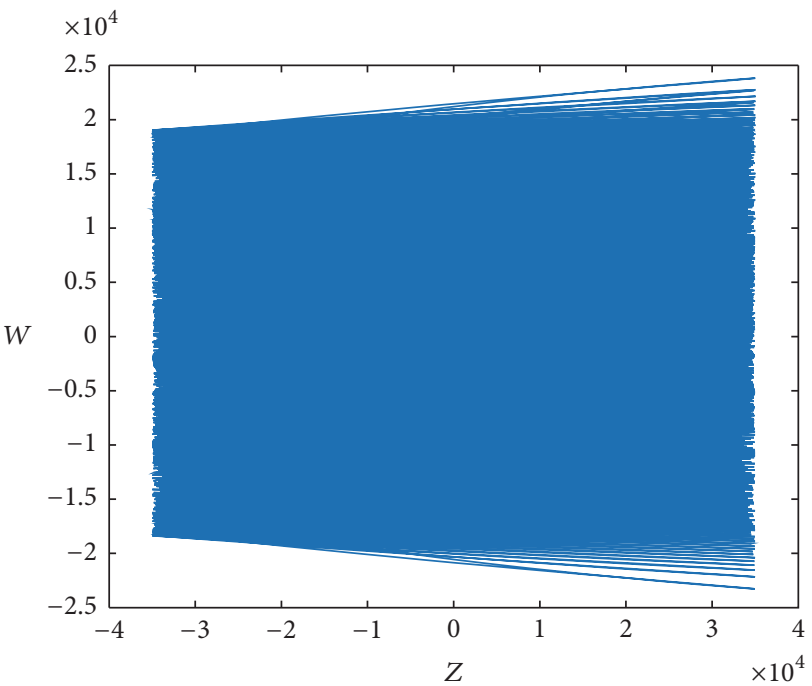

(d)

FIGURE 17: Different projections of the hyperchaotic attractor. Initial conditions: $(-3203,-26967,32052,-5434)$.

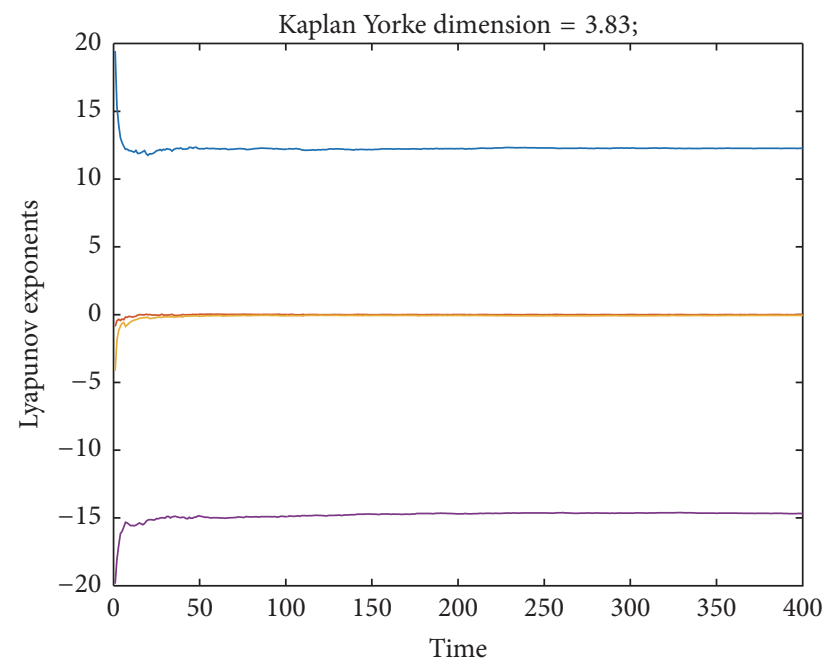

FIGURE 18: Evolution of the Lyapunov exponents values versus the integration time.

sections generate a hyperplane defined by the normal vector $\vec{n}=(0,0,0,1)$ and the point $p(0,0,0,2)$ (see Figure 19).
As can be seen in Figure 17 and as is shown more clearly in Figure 20, the hyperchaotic structures surround the origin, creating a hollow cavity. In this empty space, at $a=38$, a second chaotic attractor coexists with the hyperchaotic one previously described which remains for that value of parameter $a$.

In particular, this new topology exhibits chaotic behavior, with a great complexity, and develops an attractor (see Figure 21) very similar to that shown in Figure 13(a), although with a higher Kaplan-Yorke dimension, with a value of $D_{\mathrm{KY}}=$ 2.52. This dimension is high but does not exceed the value of three. In Table 5, the obtained Lyapunov spectrum for this chaotic attractor is shown. The coexistence of two complex structures in certain areas of the phase space reinforces the arguments about the complexity of this new system. To our knowledge, no other chaotic system with this property has been reported.

\section{Discussion and Conclusions}

In this article, a new hyperchaotic four-dimensional Lorenzbased system, especially designed to improve the complexity of traditional Lorenz dynamics, is numerically analyzed. 


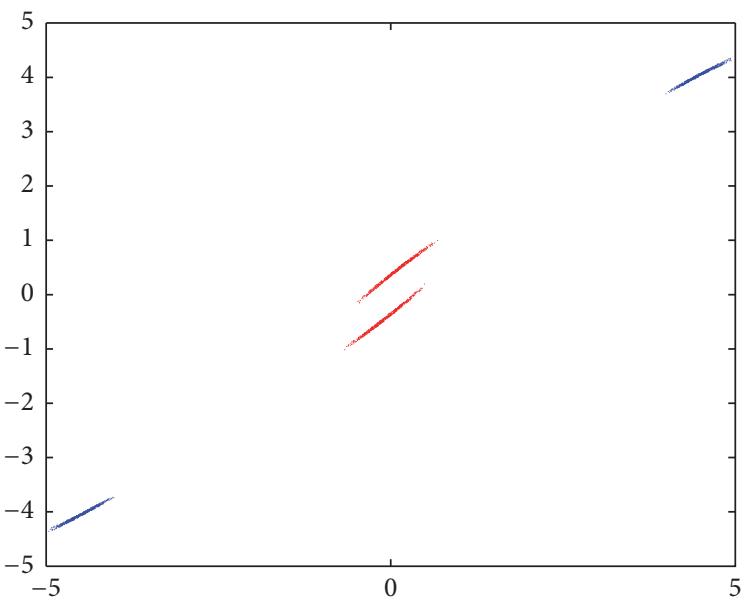

(a)

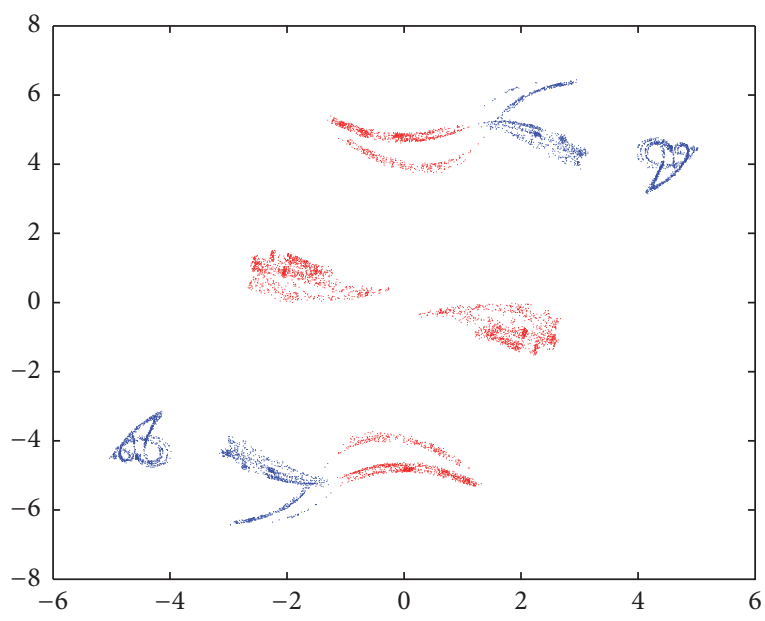

(b)

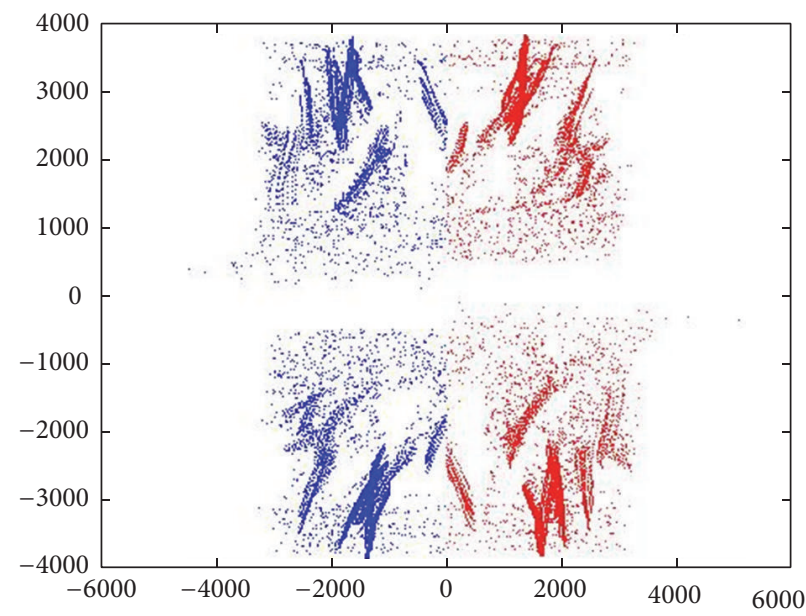

(c)

FIGURE 19: Poincaré sections of the system for different $a$ and $d$ values. Red color indicates that the flow crosses the cut plane in the sense of the normal vector. Blue color indicates that the flow crosses in the opposite sense. (a) Weak chaos with initial conditions: $(0.1,0.1,0.1,0.1)\{a=20$ and $d=10\}$. (b) Chaos with initial conditions: $(0.1,0.1,0.1,0.1)\{a=38$ and $d=2\}$. (c) Hyperchaos with initial conditions: $(-3203,-26967,32052,-5434)\{a=38.4$ and $d=2\}$.

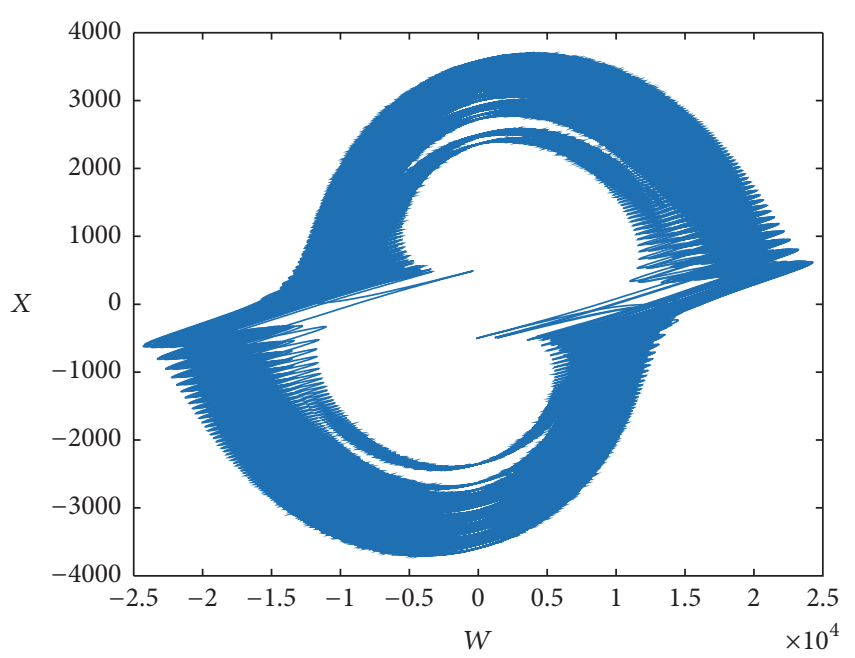

FIgURE 20: Bidimensional $X W$ projection of the hyperchaotic attractor for $\{a=38$ and $d=2\}$. Initial conditions: $(-3203,-26967$, $32052,-5434)$.
TABLE 5: Lyapunov exponents study.

\begin{tabular}{lcc}
\hline Exponent & Value & Kaplan-Yorke dimension \\
\hline$\lambda_{1}$ & 1.2092 & \\
$\lambda_{2}$ & 0 & 2.52 \\
$\lambda_{3}$ & -2.3517 & \\
$\lambda_{4}$ & -2.8622 & \\
\hline
\end{tabular}

First, a proof about the weakness of the Lorenz system, due to the amount of redundant information present in its components, is provided. Regular, chaotic, and hyperchaotic structures are located and analyzed by varying two parameters of the system. Analyses about the symmetry, stability, and bifurcation are also provided. Two Hopf bifurcations and other typical bifurcations such as period doubling bifurcations and a tangent bifurcation were observed when $a$ and $d$ control parameters were varied. Moreover, the Lyapunov exponents proved the increase in the trajectories complexity, 


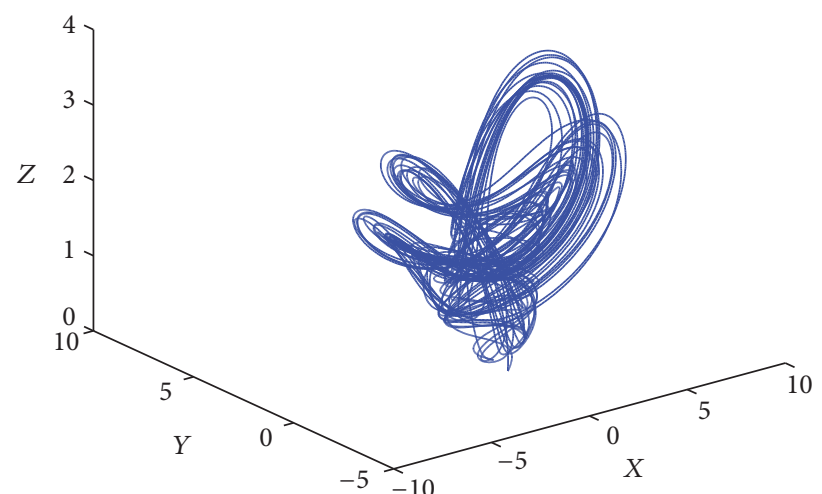

(a)

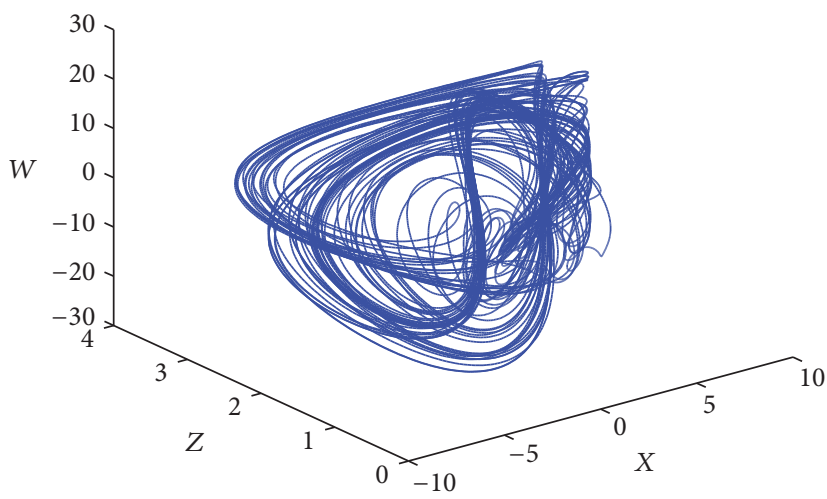

(c)

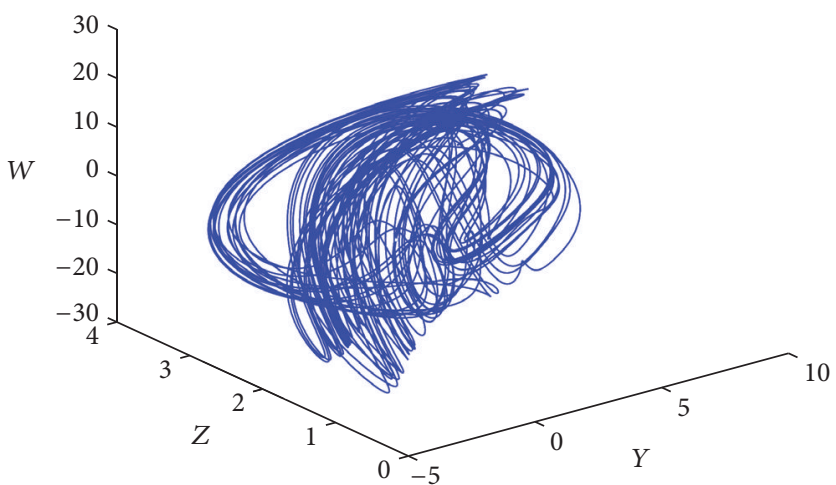

(b)

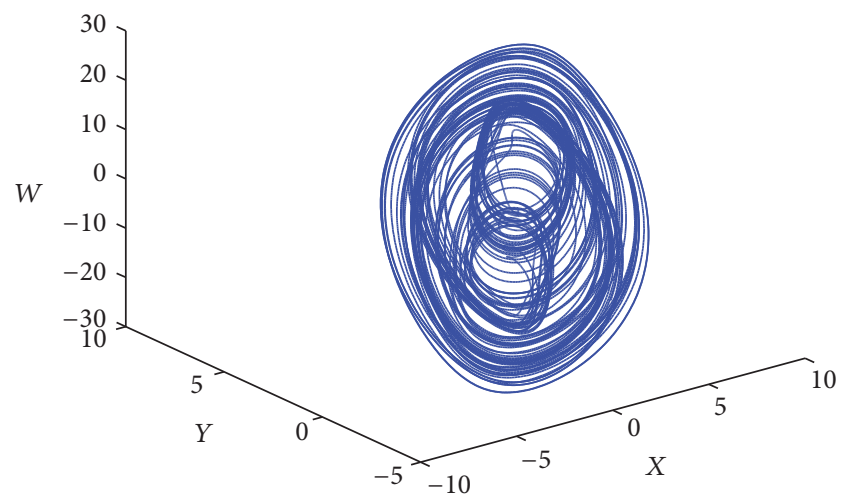

(d)

FIGURE 21: Tridimensional projections of the highly complex chaotic attractor coexisting with the hyperchaotic one. Initial conditions: $(0.1,0.1,0.1,0.1)$.

doubling in some cases the values obtained for the Lorenz dynamics. For small positive values of $d$, the third Lyapunov exponent is negative but small. This leads to a very weak contraction in this direction. On the other hand, the first exponent, which is responsible for the divergence direction, is very high. Both results lead to a Kaplan-Yorke dimension of $D_{\mathrm{KY}}=3.83$. A significant characteristic of this system is the coexistence of chaos and hyperchaos attractors as described in this paper. In addition to the chaos and hyperchaos obtained, complex chaotic-transient solutions occur in this novel system. Future work is addressed to study the coherence of this high complex system and also its application to chaos control, synchronization, and secure communications.

\section{Competing Interests}

The authors declare that there are no competing interests regarding the publication of this paper.

\section{Acknowledgments}

One of the authors, Borja Bordel, has received funding from the Ministry of Education through the FPU Program (Grant no. FPU15/03977), the Ministry of Economy and Competitiveness through SEMOLA project (TEC2015-68284-R) and from the Autonomous Region of Madrid through MOSIAGIL-CM project (Grant P2013/ICE-3019, cofunded by EU
Structural Funds FSE and FEDER). The authors are grateful for discussions with Professor Vicente Alcober.

\section{References}

[1] E. N. Lorenz, "Deterministic nonperiodic flow," Journal of the Atmospheric Sciences, vol. 20, no. 2, pp. 130-141, 1963.

[2] A. B. Orúe, V. Fernández Mármol, G. Álvarez Marañón et al., "Determination of the parameters for a Lorenz system and application to break the security of two-channel chaotic cryptosystems," Physics Letters A, vol. 372, no. 34, pp. 5588-5592, 2008.

[3] A. Ali-Pacha, N. Hadj-Said, A. M'Hamed, and A. Belgoraf, "Lorenz's attractor applied to the stream cipher (Ali-Pacha generator)," Chaos, Solitons and Fractals, vol. 33, no. 5, pp. 1762-1766, 2007.

[4] K. M. Cuomo, A. V. Oppenheim, and S. H. Strogatz, "Synchronization of Lorenz-based chaotic circuits with applications to communications," IEEE Transactions on Circuits and Systems II: Analog and Digital Signal Processing, vol. 40, no. 10, pp. 626-633, 1993.

[5] E. A. Lee, M. Niknami, T. S. Nouidui, and M. Wetter, "Modeling and simulating cyber-physical systems using CyPhySim," in Proceedings of the 12th International Conference on Embedded Software, pp. 115-124, IEEE Press, October 2015.

[6] L. M. Pecora, L. Moniz, J. Nichols, and T. L. Carroll, "A unified approach to attractor reconstruction," Chaos: An Interdisciplinary Journal of Nonlinear Science, vol. 17, no. 1, Article ID 013110, 2016. 
[7] S. Yu and W. K. S. Tang, "Tetrapterous butterfly attractors in modified Lorenz systems," Chaos, Solitons \& Fractals, vol. 41, no. 4, pp. 1740-1749, 2009.

[8] A. S. Elwakil, S. Ozoguz, and M. P. Kennedy, "Creation of a complex butterfly attractor using a novel Lorenz-type system," IEEE Transactions on Circuits and Systems I: Fundamental Theory and Applications, vol. 49, no. 4, pp. 527-530, 2002.

[9] C.-X. Liu and L. Liu, "A new three-dimensional autonomous chaotic oscillation system," Journal of Physics: Conference Series, vol. 96, no. 1, Article ID 012173, 2008.

[10] S. Yu, W. K. S. Tang, J. Lu, and G. Chen, "Generation of $n \times m-$ wing lorenz-like attractors from a modified Shimizu-Morioka model," IEEE Transactions on Circuits and Systems II: Express Briefs, vol. 55, no. 11, pp. 1168-1172, 2008.

[11] O. E. Rössler, "Chaotic oscillations: an example of hyperchaos," in Nonlinear Oscillations in Biology, vol. 17, pp. 141-156, American Mathematical Society, Providence, RI, USA, 1979.

[12] X. Liu, X. S. Shen, and H. Zhang, "Multi-scroll chaotic and hyperchaotic attractors generated from Chen system," International Journal of Bifurcation and Chaos, vol. 22, no. 2, Article ID 1250033, 15 pages, 2012.

[13] C. L. Koliopanos, I. M. Kyprianidis, I. N. Stouboulos, A. N. Anagnostopoulos, and L. Magafas, "Chaotic behaviour of a fourthorder autonomous electric circuit," Chaos, Solitons and Fractals, vol. 16, no. 2, pp. 173-182, 2003.

[14] S. Cang, G. Qi, and Z. Chen, "A four-wing hyper-chaotic attractor and transient chaos generated from a new 4-D quadratic autonomous system," Nonlinear Dynamics, vol. 59, no. 3, pp. 515-527, 2010.

[15] C.-C. Yang, "Adaptive synchronization of Lü hyperchaotic system with uncertain parameters based on single-input controller," Nonlinear Dynamics, vol. 63, no. 3, pp. 447-454, 2011.

[16] C. Han, S. Yu, and G. Wang, "A sinusoidally driven lorenz system and circuit implementation," Mathematical Problems in Engineering, vol. 2015, Article ID 706902, 11 pages, 2015.

[17] K. Sun, X. Liu, C. Zhu, and J. C. Sprott, "Hyperchaos and hyperchaos control of the sinusoidally forced simplified Lorenz system," Nonlinear Dynamics, vol. 69, no. 3, pp. 1383-1391, 2012.

[18] X. Wang and M. Wang, "A hyperchaos generated from Lorenz system," Physica A. Statistical Mechanics and Its Applications, vol. 387, no. 14, pp. 3751-3758, 2008.

[19] S. Boccaletti, J. Kurths, G. Osipov, D. L. Valladares, and C. S. Zhou, "The synchronization of chaotic systems," Physics Report, vol. 366, no. 1-2, pp. 1-101, 2002.

[20] L. M. Pecora and T. L. Carroll, "Synchronization in chaotic systems," Physical Review Letters, vol. 64, no. 8, pp. 821-824, 1990.

[21] T. Yoshida, H. Mori, and H. Shigematsu, "Analytic study of chaos of the tent map: band structures, power spectra, and critical behaviors," Journal of Statistical Physics, vol. 31, no. 2, pp. 279-308, 1983.

[22] Y. Pomeau and P. Manneville, "Intermittent transition to turbulence in dissipative dynamical systems," Communications in Mathematical Physics, vol. 74, no. 2, pp. 189-197, 1980.

[23] C. Li and J. C. Sprott, "Coexisting hidden attractors in a 4-D simplified lorenz system," International Journal of Bifurcation and Chaos, vol. 24, no. 3, Article ID 1450034, 2014.

[24] G. Baier and J. S. Thomsen, "Prototypes of attractors in four dimensions," Physical Review E, vol. 48, no. 6, pp. R4172-R4174, 1993.

[25] A. Wolf, J. B. Swift, H. L. Swinney, and J. A. Vastano, "Determining Lyapunov exponents from a time series," Physica D: Nonlinear Phenomena, vol. 16, no. 3, pp. 285-317, 1985. 


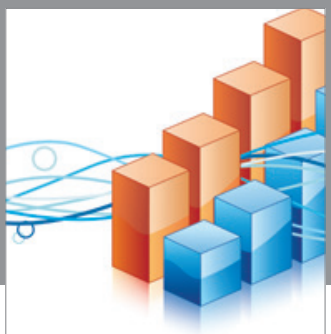

Advances in

Operations Research

vatem alat4

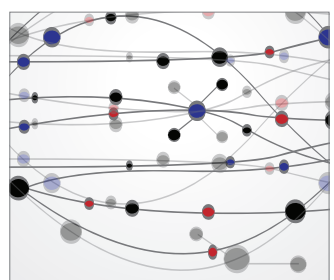

\section{The Scientific} World Journal
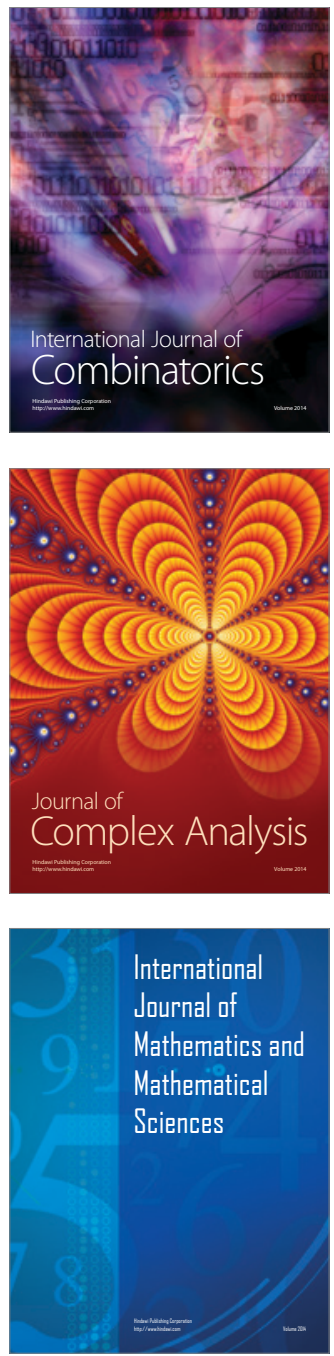
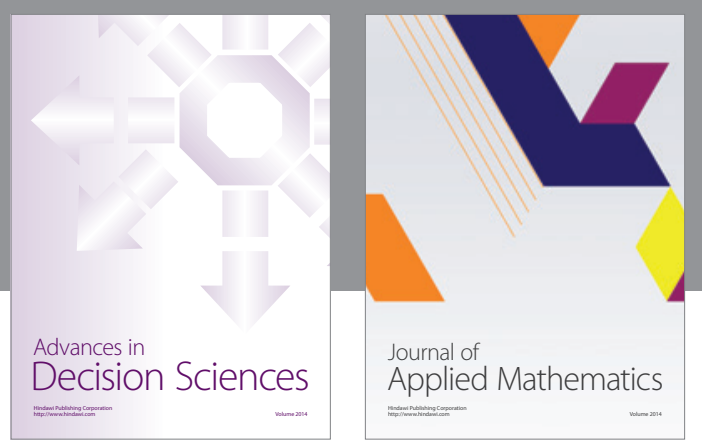

Algebra

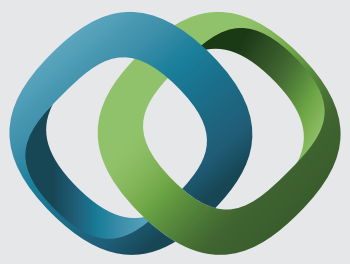

\section{Hindawi}

Submit your manuscripts at

https://www.hindawi.com
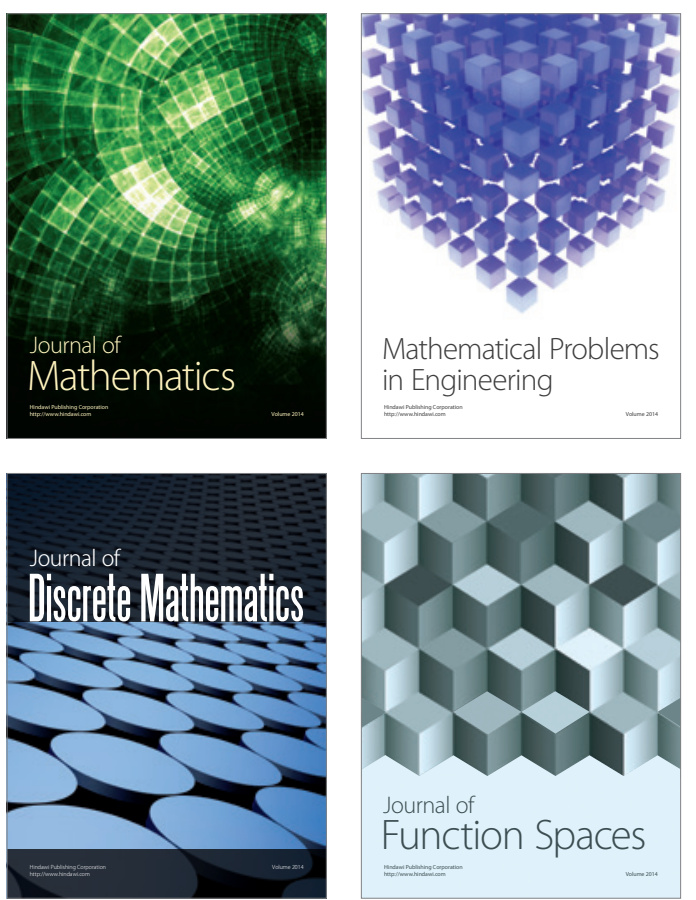

Mathematical Problems in Engineering
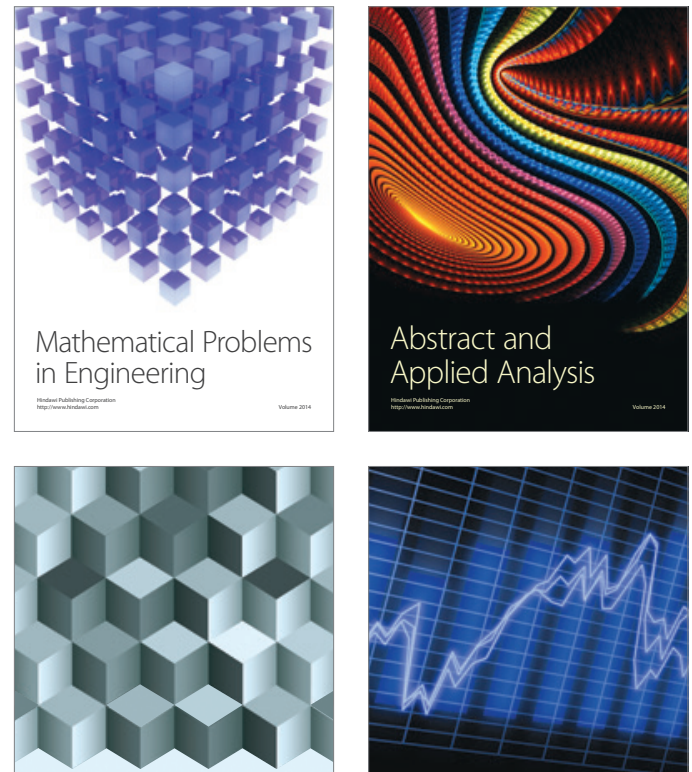

Journal of

Function Spaces

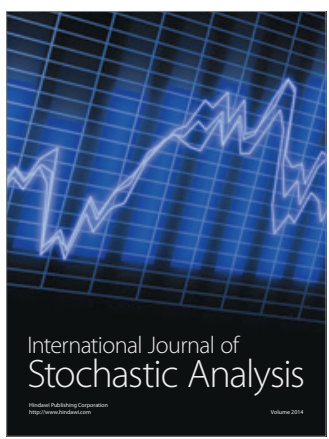

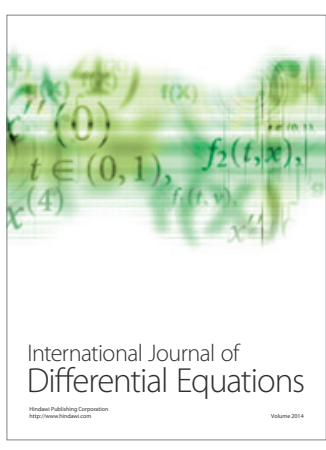
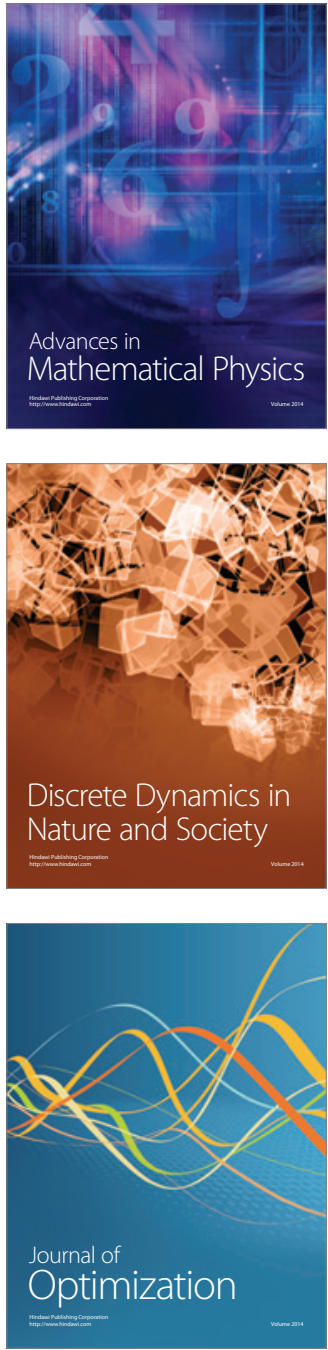